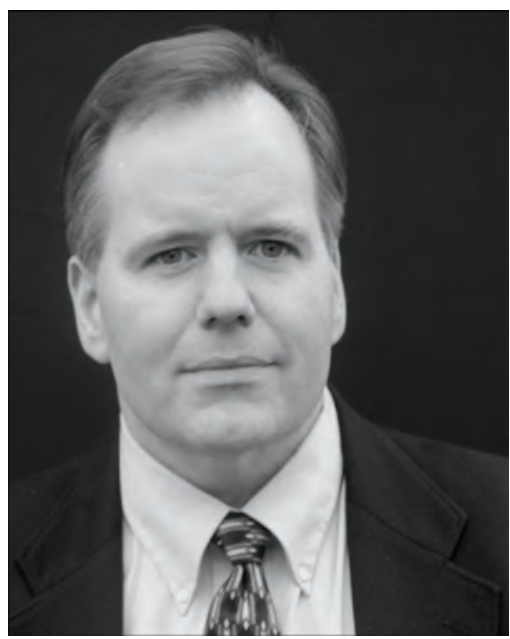

Charles King, CP

\title{
Modern research and the forgotten prosthetic history of the Vietnam war
}

Clinicians and researchers have become aware that achieving normal anatomical adduction of the femoral remnant is an important objective in transfemoral amputation surgery [1-3]. Achieving anatomical adduction has also been a long-established design goal of the transfemoral prosthetic limb [4-11]. The rationale for attaining normal anatomic adduction in the transfemoral amputee is to provide pelvic stabilization [4-6], provide efficient rest-length action of the abductor muscle group [4,8], and "reduce the lateral motion of the center of mass of the body, thus producing a smoother and more energy-efficient gait" [3]. The degree to which the prosthetic socket can influence the position of the femoral remnant has been disputed. The promotion of surgical stabilization of the femur through myodesis has been presented on the basis that the prosthesis does little to influence adduction [1-3].

In 1989, the concept that socket shape and alignment do not affect the position of the femoral remnant in transfemoral amputations was introduced into the body of prosthetic thought. In their research article, "Does socket configuration influence the position of the femur in above-knee amputation?" Gottschalk et al. concluded from observations of 50 weight-bearing X-rays of transfemoral prosthetic sockets that "no amount of lateral pressure can change the position of the femur" and stated the belief that proper anatomical adduction is achieved through specific applied surgical techniques [1]. The sockets X-rayed in Gottschalk et al.'s study comprised both quadrilateral and ischial containment socket designs, and X-ray measurements revealed a reported average of $2^{\circ}$ of abduction for all sockets surveyed. In 1989, the contention that socket shape and alignment do not influence the position of the femoral remnant was "hotly debated" at presentations at the annual meetings of the American Academy of Orthopaedic Surgeons (AAOS) and American Orthotic and Prosthetic Association (AOPA) [1,12,13 (p. 2)]. As of 2009, with numerous meeting presentations having been given and accepting literature having been published [14-15], the debate has cooled considerably. ${ }^{*}$

Two publications from the 1970s, which resulted from an X-ray checkout protocol adopted by Fitzsimons Army Medical Center (FAMC) (Figure 1) after the end of America's military involvement in Vietnam, suggest that Gottschalk et al.'s conclusion is incomplete. The publications, as they exist, can be arguably dismissed and were not cited in Gottschalk et al.'s 1989 research. They comprise a two-page technical note in a 1975 prosthetics journal [9] and an abstract published in 1977 [16]. Combined, the publications are

\footnotetext{
*Gottschalk FA. Transfemoral surgical principles. Lecture presented at Hanger Educational Fair 2009.
} Understanding Amputation Surgery; 2009 Feb 5; Reno, Nevada. 


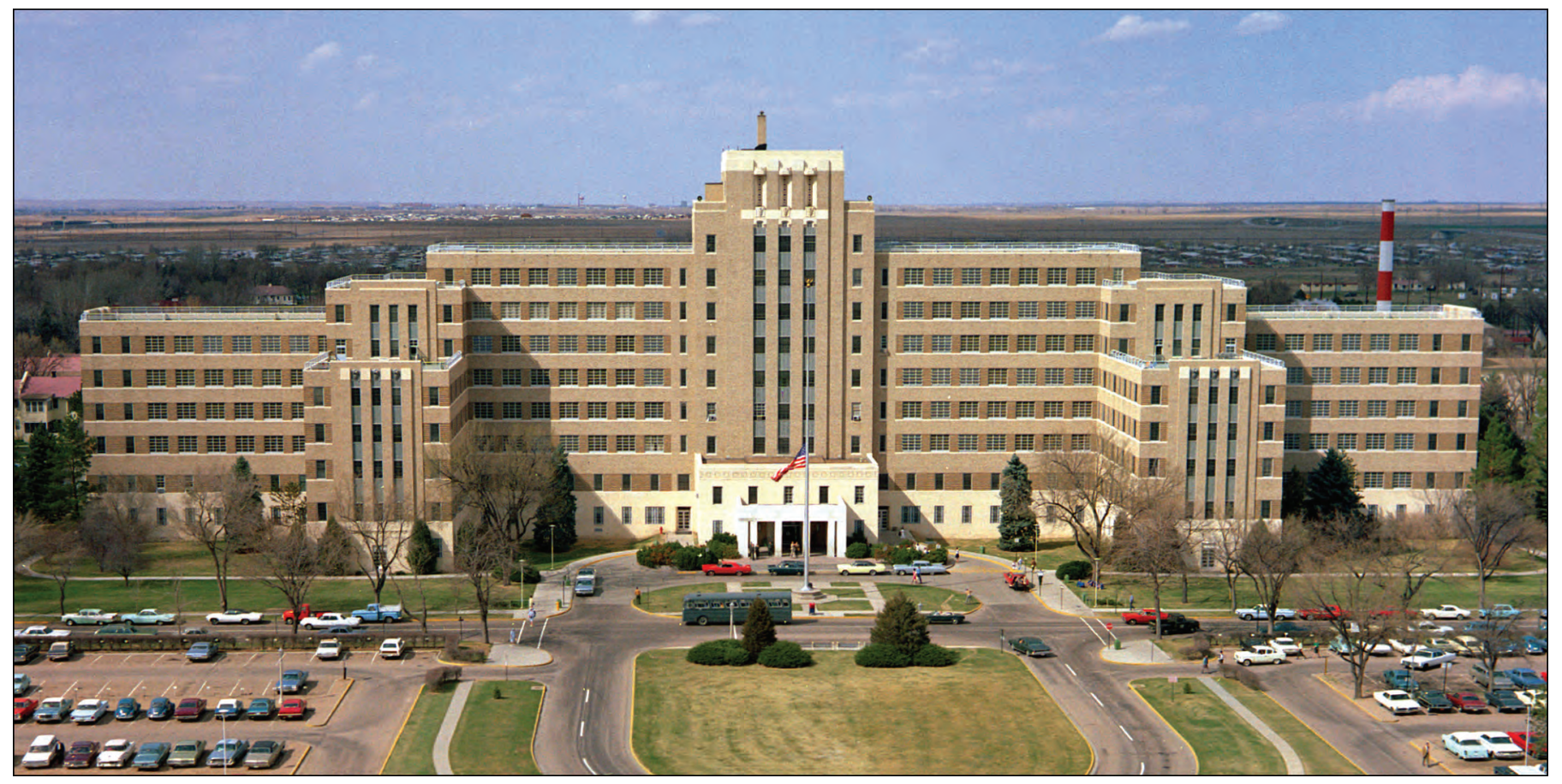

Figure 1.

Fitzsimons Army Medical Center (FAMC), located in Aurora, Colorado, circa 1970. This building, which replaced the original 1918 hospital, was dedicated on December 3, 1941, and "inactivated" on June 8, 1996 [1]. It was named after 1LT Williams Thomas Fitzsimons, Army Physician, the "first American Officer to die as a result of enemy action during World War 1” [1]. During the years 1966 to 1971, more than 600 Vietnam war amputees were treated at FAMC [2]. Source: http://www.defenseimagery.mil/imageDownload.action?guid=56f865f33d0198fb90a644bbe677a1535ce806b9. [1] Littlejohn HW, editor. Fitzsimons Army Medical Center: The Life and History: 1918-1996.Aurora (CO): Public Affairs Office, Fitzsimons Army Medical Center; 1996. [2] Brown PW. Avocational rehabilitation of amputees. Proceedings of the First International Congress on Prosthetics Techniques and Functional Rehabilitation; 1973 Mar 19-24; Vienna, Austria; 1973. p. $73-85$.

slightly more than 1,000 words. However, they serve as provenance for a collection of recently recovered documents related to FAMC's X-ray checkout of the transfemoral limb. These previously unpublished documents indicate that femoral adduction was improved, as per clinical practice, with X-ray intervention during prosthetic limb manufacture. These documents, gathered from private collections and presented in a special section of the Orthotics and Prosthetics Virtual Library, the FAMC Institutional Memory Preservation Project (http://www.oandplibrary.org/famc/), suggest that our understanding of the relationship between alignment, socket design, and femoral adduction requires further research.

The first published reference to FAMC's use of roentgenograms occurs in the second sentence of Long's "Allowing normal adduction of femur in above-knee amputations," which appeared in the
December 1975 issue of Orthotics and Prosthetics. Long wrote, "X-ray studies carried out at Fitzsimons Army Hospital since March 1974 show that very few above-knee prostheses built in the United States today achieve proper adduction of the femoral stump" [9]. It is of interest that the data from Gottschalk et al.'s research support that observation; the divergence and controversy emerge from the conclusion from that data. This 1975 technical note also introduces an eponymous bench alignment protocol known as "Long's Line" and the evolving narrow mediolateral (ML) socket design. Long wrote, "In every case, I found that, by simply realigning the knee and foot with respect to the socket, the amputee could bring the femur into a normal position" [9]. The socket design then evolved to achieve the design goal of anatomical adduction as verified by X-ray. Providing the rationale of the emerging narrow $\mathrm{ML}$ 
socket design, Long wrote, "Adduction is difficult to maintain when the ML dimension of the socket is excessive, because support of the femur is lost when weight is applied and the top of the socket moves laterally" [9]. The goal of maintaining proper alignment necessitated the changes in socket design. An early surviving image, scanned from recovered $16 \mathrm{~mm}$ film, of a patient's prosthesis manufactured before and after the employment of X-rays at FAMC shows a striking change in appliance shape (Figure 2).

Thirteen years prior to Gottschalk et al.'s published research, Mayfield presented a paper titled "A new look to and through the above knee prosthesis" at the 1977 AAOS annual meeting [17]. The abstract of this presentation was published in Orthopedic Transactions (a collection of medical abstracts) by the Journal of Bone and Joint Surgery [16]. Scanlon and Long are listed as coauthors. The abstract details the aggregate $\mathrm{X}$-ray measurement data of 71 weightbearing X-rays taken of 51 transfemoral amputees [16] at FAMC in Aurora, Colorado [18].

Mayfield's abstract contains two main groups of 38 and 13 patients, respectively. Within the group of

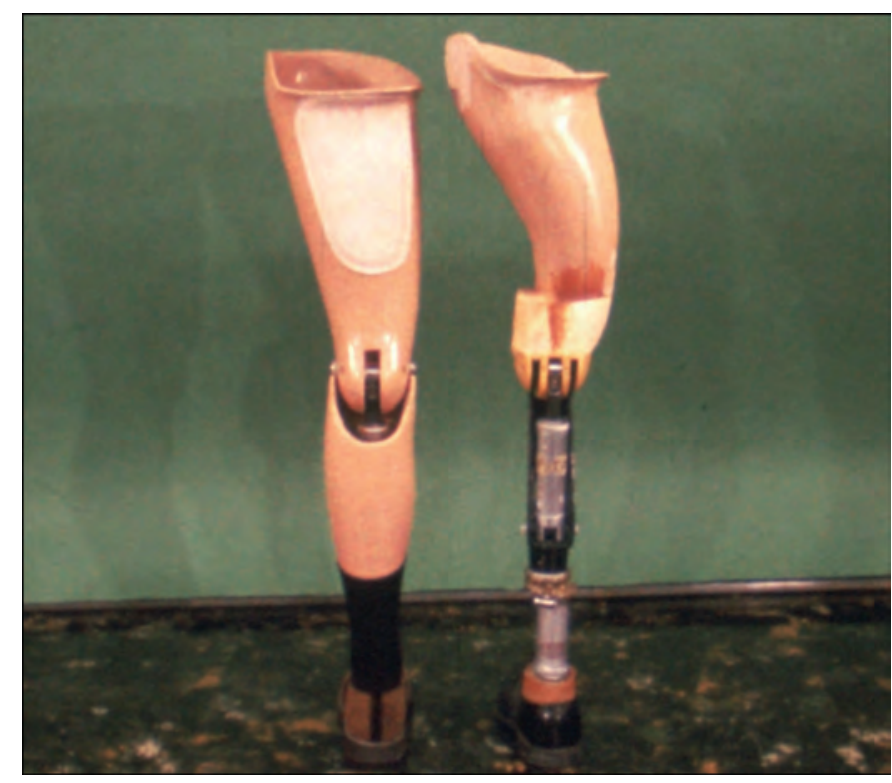

Figure 2.

Two months after adoption of the X-ray checkout of the transfemoral limb at Fitzsimons Army Medical Center, a manifestly different prosthesis look evolved. Left: Prosthesis manufactured before X-ray evaluation. Right: Prosthesis manufactured with X-ray evaluation. Source: http://www.oandplibrary.org/famc/photos/.
38 X-rayed patients, femoral abduction was discovered in the majority of the standard-aligned quadrilateral sockets. Twenty of these patients were provided with a replacement limb using "revised fitting techniques” and X-rays were again taken [16]. Figure 3 shows a patient prior to X-ray checkout at FAMC. Depicted in Figure $\mathbf{4}$ is a surviving example of the early comparative FAMC X-rays as mentioned in Mayfield's 1977 abstract. It should be noted that although no crosswalk document exists that connects the recovered FAMC X-ray images and Mayfield's patient population, undoubtedly

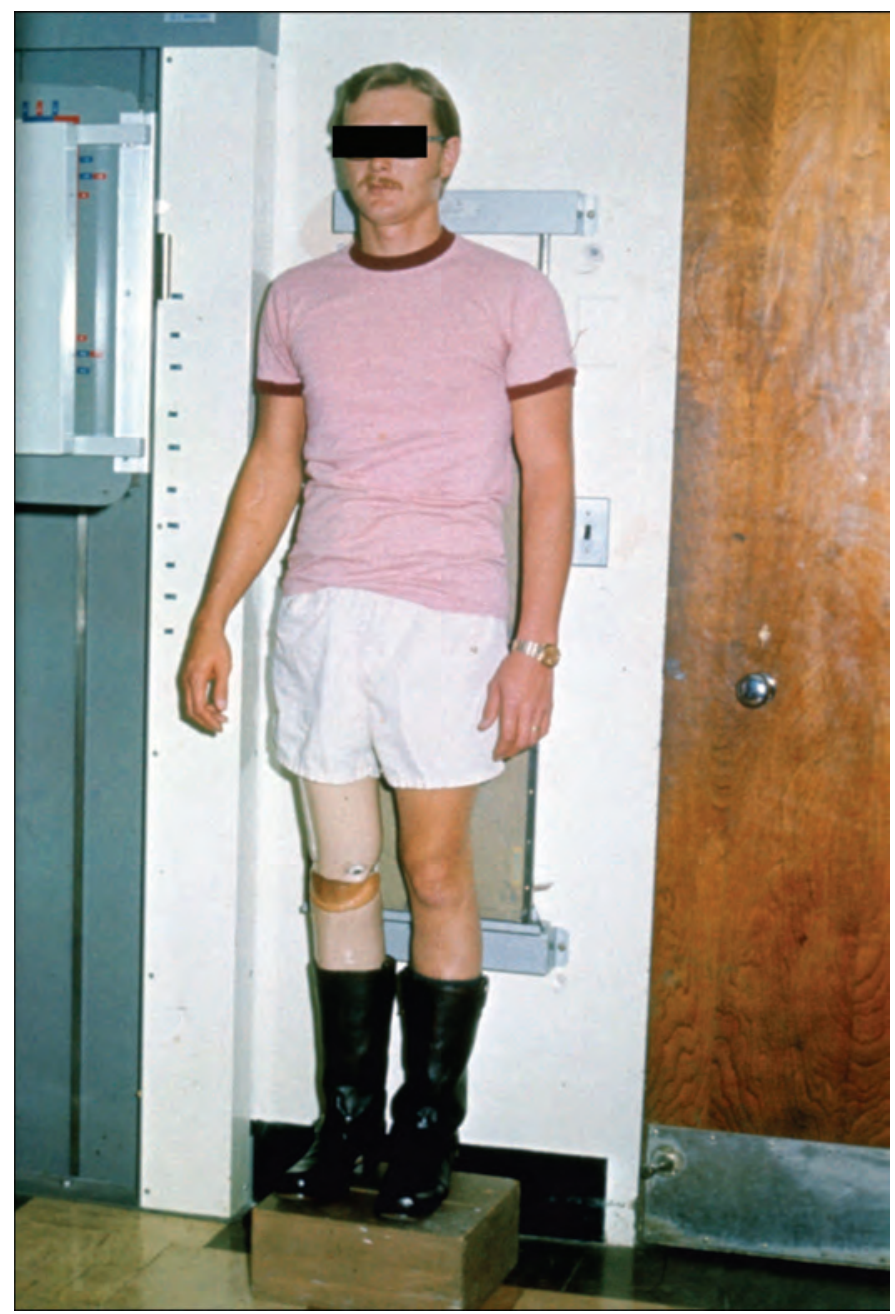

Figure 3.

Transfemoral amputee at Fitzsimons Army Medical Center, circa January 1976, positioned in front of a $14 \times 36$ in. cassette for radiographic evaluation of his prosthesis. Source:

http://www.oandplibrary.org/famc/photos/. 
some of Mayfield's study participants are represented in these recovered X-rays.

Referring to Figure 4, an improvement of the adduction angle of the femoral remnant can be observed in the revised alignment prosthesis. Mayfield wrote that each of the patients who received a revised alignment limb "had a significant improvement in the adduction of the hip in the socket, improved lateral wall support and improvement in gait.” [16] Long's clinical observation from his 1975 article was that the revised "alignment permits the AK [above-knee] amputee to walk freely, securely, and without back pain, and side motion of trunk is greatly stabilized” [9].

Scanlon (physical therapist at FAMC), in a taped interview, provides his recollection of the revised alignment limbs provided to FAMC patients:

It was a dramatic, almost immediate change in their walking. Their gluteus medius limp and their Trendelenburg would certainly be much

(a)

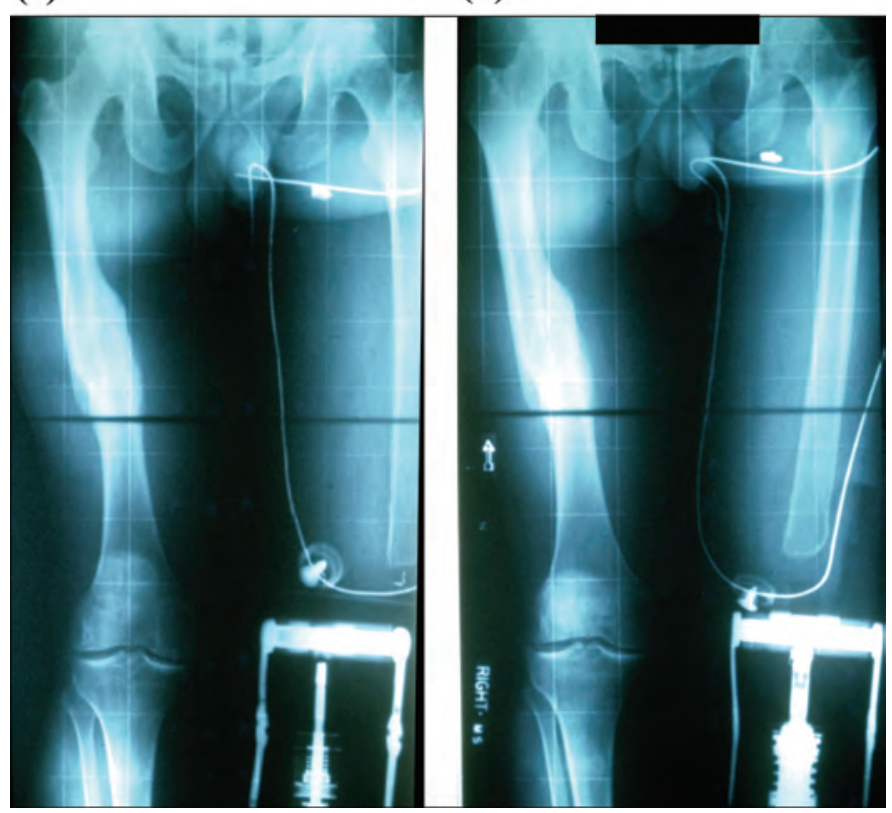

Figure 4.

(a) Fitzsimons Army Medical Center (FAMC) X-ray survey of standard alignment quadrilateral limb, date unknown. (b) FAMC X-ray checkout of revised alignment prosthesis, September 1975. Note the change in alignment and socket shape resulting in improved femoral adduction. Source: http://www.oandplibrary.org/famc/photos/. less. The patients would make comments about how the new prosthesis and the alignment felt much more secure and much more balanced. It is something the patients would verbalize almost immediately when they put the new prosthesis on. It wasn't something that took days or weeks of training before they could see the difference or feel the difference. It seemed to be almost immediate.

The "revised fitting technique" referenced in Mayfield's abstract would eventually evolve into what has come to be called the ischial containment narrow ML socket [16]. The illustrations in Figure 5 are republished from Mayfield's 1977 abstract and presented in reverse order from the original [16]. They schematically represent the femoral abduction discovered (Figure 5(a)) in the X-rayed quadrilateral sockets and the improvement in adduction achieved and documented through alignment and socket shape modification (Figure 5(b)).

The 1977 abstract provides further detail of the emerging narrow ML socket design first referenced in Long's 1975 technical note. Mayfield writes,

. . . a revised fitting technique was devised contouring a slightly convex inward lateral wall to support the femur against lateral thrust and emphasizing hip adduction during socket manufacture. . . . The AP [anteroposterior] socket dimension is increased to accommodate soft tissue displacement as the ML dimension is decreased. The knee/ shank/foot unit is displaced lateral to the distal end of the socket and as the foot is brought toward the midline, hip adduction is assured [16].

The second set of X-rayed amputees in Mayfield's study consisted of 13 patients. The abstract reports that these " 13 previously unfitted new amputees were fitted by the revised techniques and X-rays revealed adduction of the hip in all cases" [16]. This result suggests that the prosthetic socket and alignment

* Recorded interview, Jim Scanlon, PT: 08/14/07. Revised by Jim Scanlon 10/05/09. 


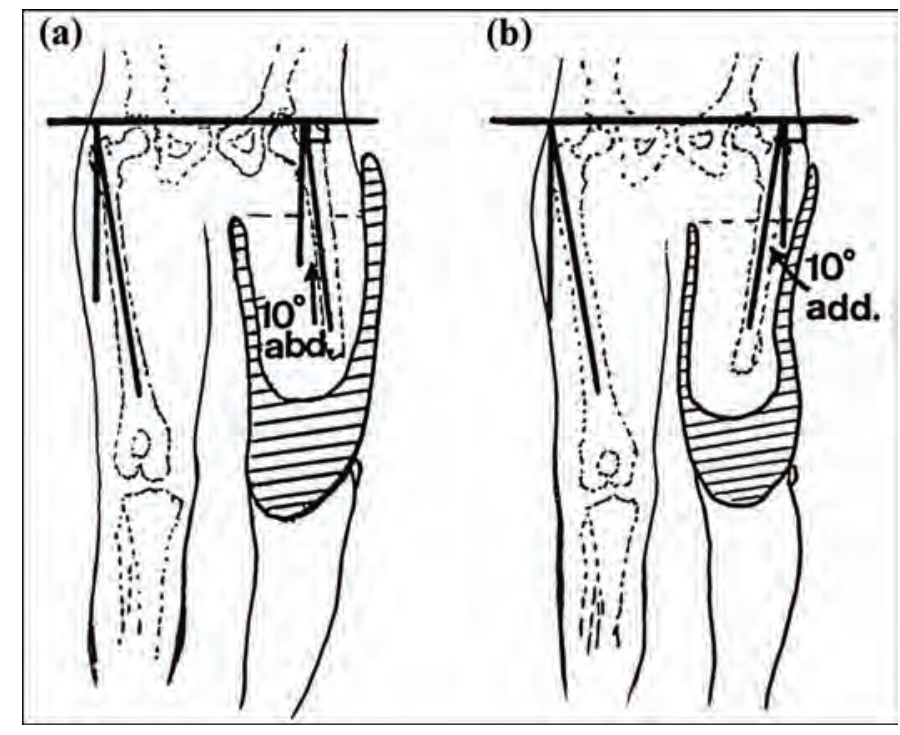

Figure 5.

(a) Diagram of abduction (abd.) discovered by survey X-rays of transfemoral prostheses taken at Fitzsimons Army Medical Center (FAMC). (b) Diagram of improved transfemoral adduction (add.) achieved at FAMC with alignment and socket modifications as verified by checkout $\mathrm{X}$-ray. Modified from the original source: Mayfield GW, Scanlon J, Long I. A new look to and through the above knee socket. Orthop Trans. 1977;1(1):95. Available from: http://www.oandplibrary.org/famc/?linkto=abnewlook\#abnewlook.

techniques developed working from X-rays taken at FAMC were effective and repeatable in achieving support for the amputated femur in adduction.

In contrast to the FAMC study, no changes were undertaken in socket design or alignment in the 1989 research of Gottschalk et al. All conclusions were limited to X-ray survey alone. In Gottschalk et al.'s study, 23 patients with ischial containment sockets and 27 patients with quadrilateral sockets were X-rayed under weight-bearing conditions with the adduction/ abduction angles of the femoral remnant recorded. An analysis of variance of the quadrilateral and ischial containment X-ray measurements, which revealed no statistically significant variance between the two groups (they were reported as having identical means), is referenced in Gottschalk et al.'s article. Both socket styles revealed a reported average of $2^{\circ}$ of abduction. This finding went against the fundamental tenet of the ischial containment (narrow ML) socket designnamely, improved femoral adduction-and led to Gottschalk et al.'s conclusion that the femur is not influenced by either the type of socket or the alignment of the prosthesis. The question posed by Gottschalk et al.'s article title, “Does socket configuration influence the position of the femur in above-knee amputation?" was answered in the negative. A longer selection from Gottschalk et al.'s article puts a previously quoted line in context:

The muscle envelope surrounding the femur cannot be displaced by external forces. Once the femur is in an abducted position within the muscle compartment, no amount of lateral pressure by the prosthesis will alter the existing configuration. In the majority of cases that we examined, the residual femur was not in the normal anatomically adducted position. The socket configuration did not affect the position of the femur within the socket [1].

The first two sentences of this quotation are not supported by evidence presented in Gottschalk et al.'s article. These sentences are provided without citation, support, or empirical evidence. No attempt was ever undertaken in Gottschalk et al.'s research to determine whether a modified socket configuration could change the position of the femur. This omission limits the validity of the conclusion regarding socket design and alignment when applied to all transfemoral sockets. The research did not involve addressing the effect or influence of alignment and socket design on the femur. With respect to the angular orientation of the femoral remnant, prosthetic influence was not established or explored. Again, no artificial limbs were modified during the research to validate if, in actual fact, the position of the femur is forever unchanged by alignment or socket design.

In 1994, Gottschalk and Still's article, "The biomechanics of trans-femoral amputation," was published in the journal Prosthetics and Orthotics International [2]. They write (with the 1989 literature cited therein), "It is not possible to hold the residual femur adducted with a prosthetic socket irrespective of its shape or design, as has previously been reported (Gottschalk et al., 1989) since the femur cannot be displaced in its soft tissue envelope" [2]. This citation suggests that the conclusion drawn from Gottschalk et 
al.'s X-ray research is being applied to all prosthetic sockets. As the 1989 research did not involve addressing the effect or the influence of socket design or alignment on the femur, this is an incomplete conclusion. Gottschalk et al.'s prosthetic findings, like Long's 1975 quoted report of FAMC's X-ray research findings, revealed a failure to achieve design goals as reduced to actual prosthetic practice.

The U.S. Army's 1998 Rehabilitation of the Injured Combatant, Volume 1, states “. . . that the [ischial containment] narrow medio-lateral socket does not provide improved femoral adduction control, and that this control does require surgical reattachment of the adductor musculature for this to occur" [14]. The sole cited reference for this assessment is Gottschalk et al.'s 1989 article. In Gottschalk et al.'s 1989 cited study, a "biomechanical model" of the stump was put forth in which the adductor muscles of a transfemoral amputee were both compromised and exhibited a disadvantageous length-tension relationship after amputation surgery [1]. This allowed the femur to move into abduction "because of the relatively unopposed action of the abductor system” [1]. Gottschalk et al. devised a surgical technique to ensure proper femoral adduction in a transfemoral residual limb, proposing "for the surgeon to actively overcorrect the alignment" of the femur [1]. They stated that "successful prosthetic fitting starts at the time of surgery” [1]. Mayfield, referencing femoral remnant myodesis, observed in his chapter in Orthopedic Surgery in Vietnam that "most of the traumatictype amputations sustained in the war zone were not amenable to this procedure" [19]. Adding clarification, Mayfield related that myodesis of the "residual adductors is important in helping maintain hip adduction, if those adductors are present and in good shape. Frequently this was not the case in our [Vietnam] amputees."* The previous quote from Rehabilitation of the Injured Combatant, Volume 1, is not an unreasonable encapsulation of the 1989 research relating to the ischial containment narrow ML socket, which in Gottschalk et al.'s study clearly did not dem-

*Personal communication, Jerry Mayfield, MD: 01/07/07. onstrate improved femoral adduction. It is, however, limiting to assert that surgery is the only means to improve adduction. It would appear that the practical lessons learned during the Vietnam war have not been transferred to peacetime scholarship.

Mayfield's abstract was never followed up with contemporaneous publication of the complete study. The paper was written in a condensed form [20] for a 12-minute AAOS presentation [17,20], with the intention-never realized - of expanding it into a more comprehensive research paper [20]. A surviving copy of the paper has recently been discovered. Figure 6 depicts the original recovered pages from Mayfield's research paper that contain the individual patient adduction/abduction measurements. The recovered data are consistent with the statistics presented in the 1977 abstract published in Orthopedic Transactions. Scanlon and Long are cited as coauthors in both Mayfield's abstract and the recovered paper. Mayfield explains that he was the author of both "independent from any direct manuscript input by the coauthors," and credited his coauthors "based on multiple collaborative discussions concerning the subject” [18]. With respect to the new socket design or "revised fitting techniques" [16] involved in the study, Mayfield reports, "it was Ivan [Long] who came up with the methods of constructing a better socket (increase AP dimension, decreased ML dimension, and a convex inward lateral)." ${ }^{\dagger}$

The 12-page paper has been digitally retypeset and republished (along with the original source document) in a peer-reviewed history section of the Orthotics and Prosthetics Virtual Library that contains documents preserving FAMC's institutional memory relating to the treatment of Vietnam war amputees (http://www.oandplibrary.org/famc/.) [21]. Given the relative scarcity of prosthetic X-rays, these newly recovered evaluations are a valuable body of data available for analysis and interpretation.

The X-ray adduction/abduction measurement methodology, devised at FAMC in the 1970s, is graphically illustrated in Figure 7. Anteroposterior

\footnotetext{
${ }^{\dagger}$ Personal communication, Jerry Mayfield, MD: 03/05/07.
} 


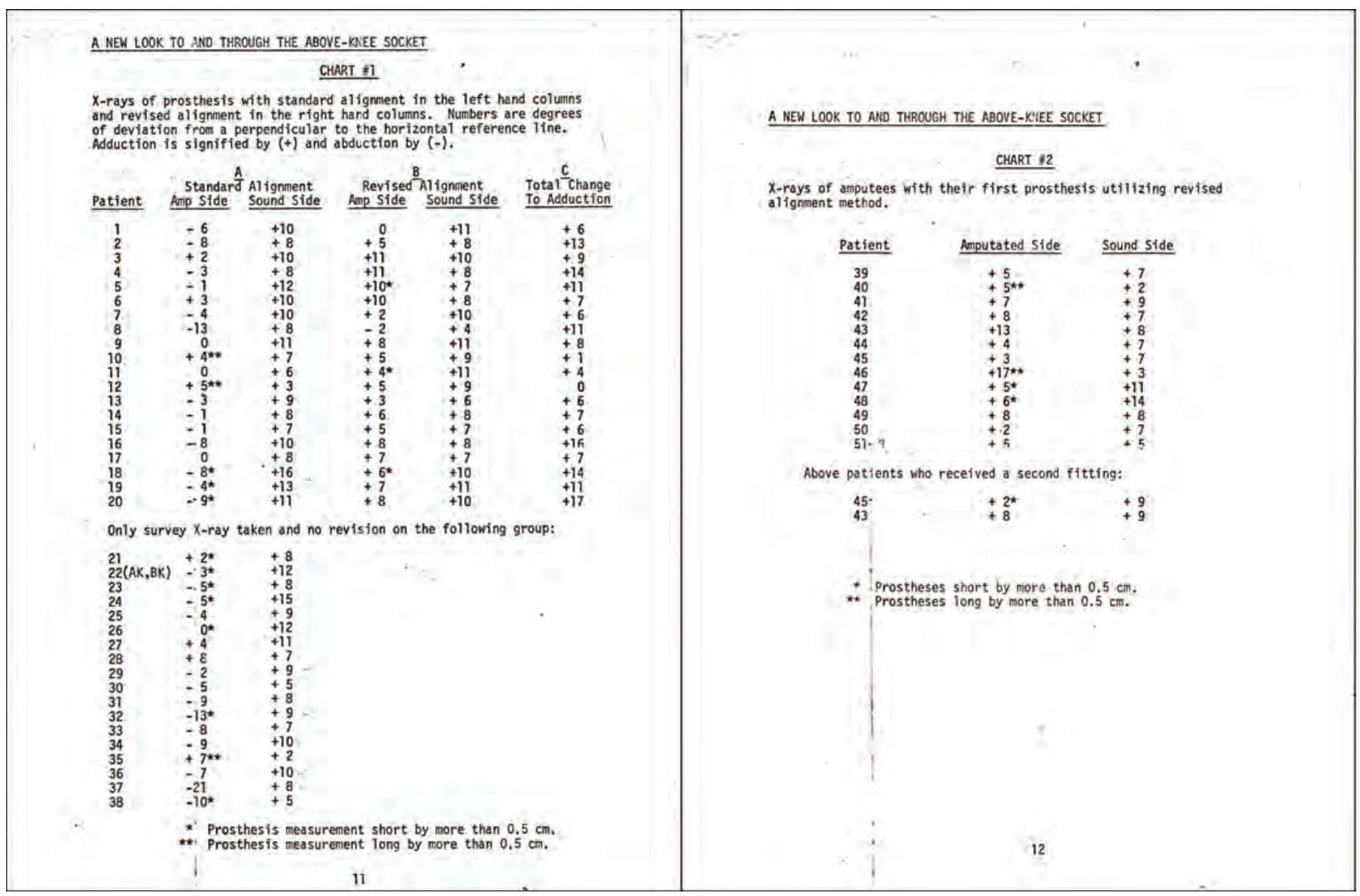

Figure 6.

Page 11 and 12 of Mayfield's original recovered research paper, comprising two charts of 73 X-rays taken of 51 transfemoral amputees treated at Fitzsimons Army Medical Center. Source: Mayfield GW, Scanlon J, Long I. A new look to and through the above-knee socket (Recovered research paper) [Internet]. Gainesville (FL): Digital Resource Foundation for the Orthotics and Prosthetics Community, The Orthotics and Prosthetics Virtual Library, Fitzsimons Army Medical Center Institutional Memory Preservation Project; 2009 Jun 22 [cited 2009 Jun 30 ]. Available from: http://www.oandplibrary.org/famc/supportdocuments/?linkto=anewlook.

X-rays were taken with the "heels 2 inches apart, with the weight distributed equally on the prosthetic and normal extremity" [16]. A "horizontal reference line" is placed across the femoral heads in the X-ray, and a "perpendicular" is drawn at the point where a line, projected along the axis of the femurs, intersects [21]. Adduction is indicated by positive numbers and abduction is indicated by negative numbers [21]. The use of negative numbers allows for a mathematical average of the measurements to be calculated. The exact measurement methodology employed in Gottschalk et al.'s study is not documented; a subsequent literature reference suggests that the measurements of femoral angles were most likely taken from vertical lines perpendicular to a level floor [22].

Similar to the published abstract, Mayfield's recovered research paper presents the individual adduction/abduction angle measurements taken from weight-bearing X-rays of 51 patients and their 73 different sockets (which represent an additional 2 sockets than referenced in the abstract). Figure 6 depicts the recovered study's patient statistics pages as recorded in two typed charts in Mayfield's recovered study. Chart \#1 contains data from 38 patients Xrayed in their "standard alignment" quadrilateral sockets. Twenty patients (numbered 1-20 in Chart \#1) received limbs with the quadrilateral standard alignment as well as replacement limbs with a "revised 


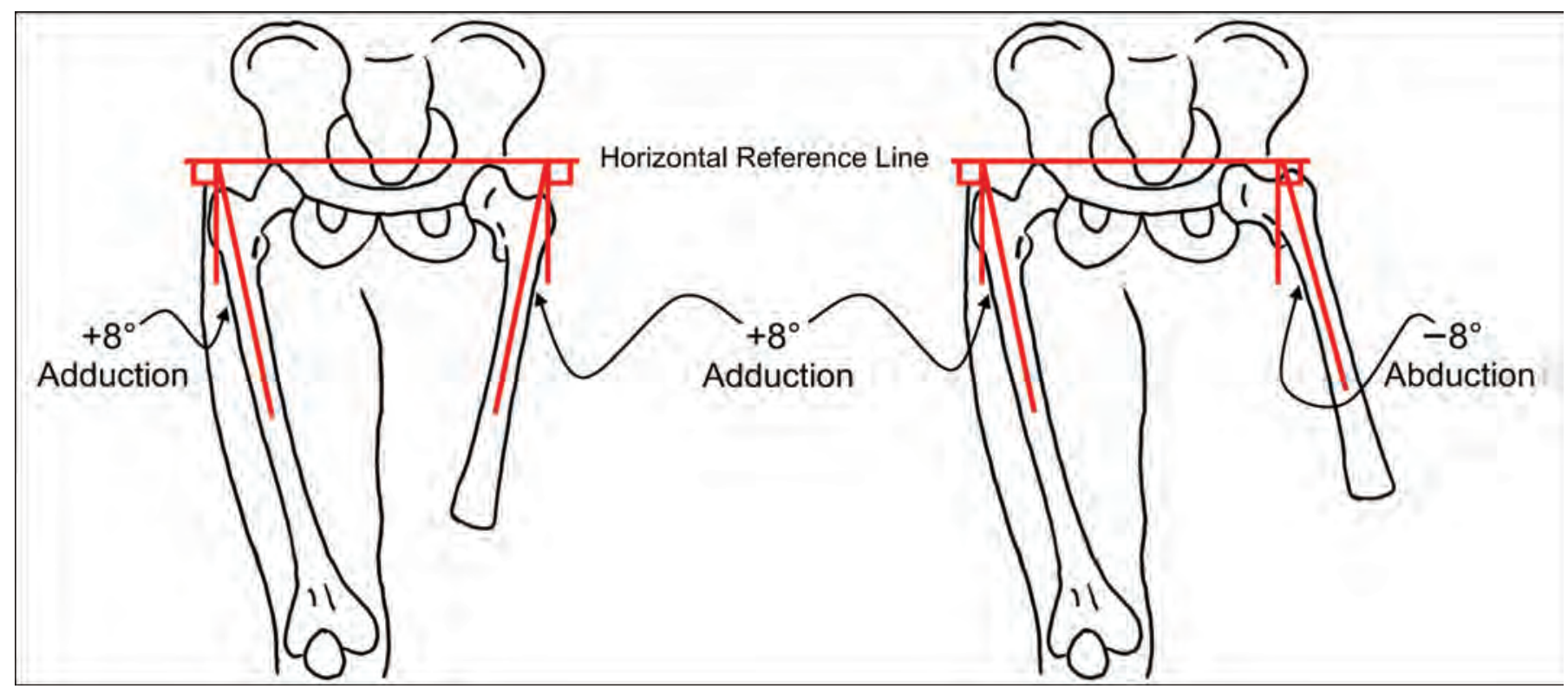

Figure 7.

Mayfield's transfemoral amputee X-ray measurement methodology for recording the angular position of the femoral remnant. Adduction is indicated by positive numbers and abduction by negative numbers.

alignment” [21]. Eighteen patients (numbered 21-38 in Chart \#1) only received limbs with the quadrilateral standard alignment. Chart \#2 contains data from 13 patients (numbered 39-51) who received only the "revised alignment" prosthesis; two of these patients had an X-rayed "second fitting" [21]. The groupings found in Mayfield's study represent the chronological prosthetic treatment of the amputee patient population at FAMC and the evolution of the revised socket alignment technique. Mayfield's data set has been resorted into two groups. The descriptive statistics are presented in Table 1 (Microsoft Excel 2002, Microsoft Corp; Redmond, Washington). In Mayfield's recovered study, the quadrilateral socket group, Group 1, has an average of $3.6^{\circ}$ of abduction and the revised alignment socket group, Group 2, which has been labeled Proto-IC (protoischial containment), achieved an average of $6.2^{\circ}$ of adduction. The revised alignment sockets X-rayed in Mayfield's study have been labeled Proto-IC because they represent limbs made with Long's Line alignment and can be considered the origin of what eventually became classified as ischial containment or ischial ramal containment (IRC) socket design [23-24]. In the prosthetics field,
Table 1.

Femoral remnant measurement statistics of Mayfield's quadrilateral and protoischial containment groups. Positive numbers represent adduction and negative numbers abduction.

\begin{tabular}{lcc}
\hline Variable & $\begin{array}{c}\text { Quadrilateral Group 1 } \\
\text { Femoral Remnant } \\
(\boldsymbol{n}=\mathbf{3 8})\end{array}$ & $\begin{array}{c}\text { Protoischial } \\
\text { Containment Group 2 } \\
\text { Femoral Remnant } \\
(\boldsymbol{n}=\mathbf{3 5 )}\end{array}$ \\
\hline Mean & -3.6 & 6.2 \\
Median & -3.5 & 6.0 \\
SD & 6.0 & 3.7 \\
Minimum & -21.0 & -2.0 \\
Maximum & 8.0 & 17.0
\end{tabular}

$\mathrm{SD}=$ standard deviation.

Data source: Mayfield GW, Scanlon J, Long I. A new look to and through the above-knee socket (Recovered research paper) [Internet]. Gainesville (FL): Digital Resource Foundation for the Orthotics and Prosthetics Community, The Orthotics and Prosthetics Virtual Library, Fitzsimons Army Medical Center Institutional Memory Preservation Project; 2009 Jun 22 [cited 2009 Jun 30]. Available from: http:// www.oandplibrary.org/famc/?linkto=newlookre\#newlookre.

the socket classification has also been interchangeably called narrow ML or the ischial containment narrow ML socket design as a means of differentiation from the standard quadrilateral design. 
Figure 8 is a scatter plot graph showing Mayfield's X-ray statistics (GraphPad Prism, version 5.0, GraphPad Software; San Diego, California). The red bands in the scatter plot graphically show the standard deviation, and the bisecting yellow bars indicate the group's mean. Note the $9.8^{\circ}$ difference between the mean of the Proto-IC sockets and the mean of the quadrilateral sockets.

Mayfield's quadrilateral sockets' mean reveals $1.6^{\circ}$ greater abduction than Gottschalk et al.'s reported findings, which is likely an insignificant statistical difference. Mayfield's Proto-IC sockets' mean shows an $8.2^{\circ}$ difference toward adduction compared with Gottschalk et al.'s reported measurements for the ischial containment socket. The divergence of the group means in Mayfield's recovered data would suggest that Gottschalk et al.'s conclusion that femoral adduction is not influenced by socket design or alignment [1-2] is an incomplete assessment.

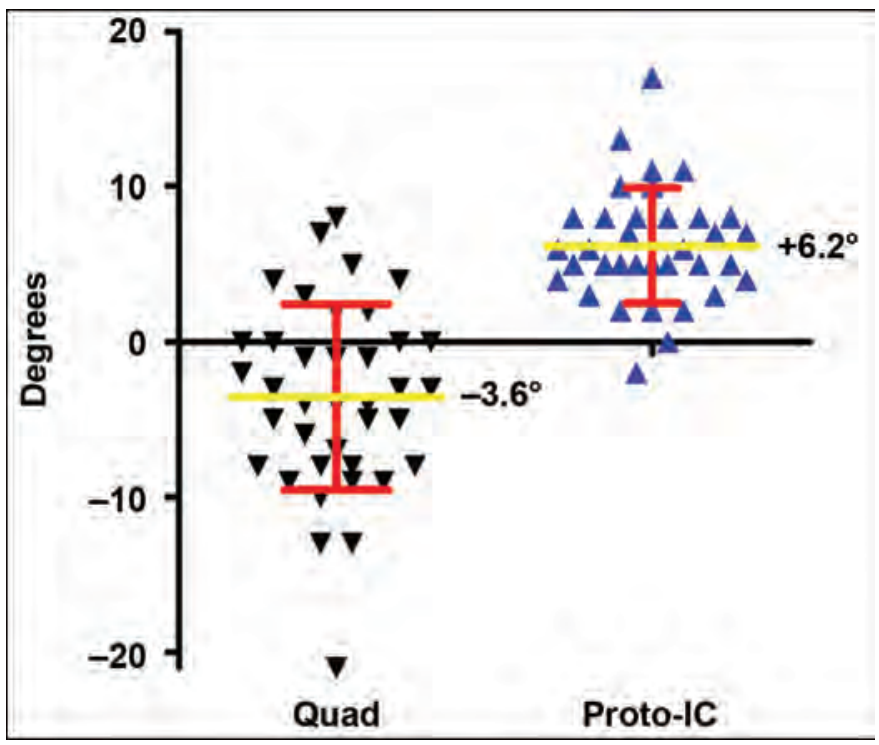

Figure 8.

Scatter plot of Mayfield's recovered transfemoral X-ray femoral remnant adduction/abduction measurements. Vertical red bands represent standard deviation and bisecting yellow bars indicate the group's mean. Quad = quadrilateral $(n=38)$, Proto-IC = protoischial containment $(n=35)$. X-ray data source: Mayfield GW, Scanlon J, Long I. A new look to and through the above-knee socket (Recovered research paper) [Internet]. Gainesville (FL): Digital Resource Foundation for the Orthotics and Prosthetics Community, The Orthotics and Prosthetics Virtual Library, Fitzsimons Army Medical Center Institutional Memory Preservation Project; 2009 Jun 22 [cited 2009 Jun 30]. Available from: http://www.oandplibrary.org/famc/ supportdocuments/?linkto=anewlook.
Mayfield's data noted leg-length discrepancies in the recorded patient statistics without corrections made in the study. The recovered paper states that the "discrepancies were accepted on the basis the angles would be similar to those in the patient functioning with leg-length discrepancies” [21]. In contrast with the textual account of the data in the recovered text, 34 percent of the quadrilateral sockets (13) and 23 percent of the revised alignment Proto-IC sockets (8) (21 limbs in total) were recorded as either too long or too short by $0.5 \mathrm{~cm}$ (3/16 in.). The X-ray measurement methodology employed at FAMC, as depicted in Figure 7, involves a horizontal reference line drawn across the tops of the femoral heads such that the collected measurements would be specifically influenced by leg-length inequalities. Mayfield writes, "Shortening of the prosthesis tends to increase the angle of the hip abduction on the amputated side . . . due to the pelvic tilt increasing the angle of adduction on the sound side. The converse is true if the prosthesis is too long, causing a pelvic tilt in the opposite direction and increasing the angle of adduction on the amputated side” [21]. In Gottschalk et al.'s article, equal length was reported as being confirmed before $\mathrm{X}$-rays, but no reference is made in the study regarding how corrections were made for any length differences encountered [1]. Had the floor been established as the horizontal reference line, which was subsequently recommended by Gottschalk [22], with perpendicular lines extended, intersecting with the long axis of the femora, any leg-length discrepancies would have had an equal but opposite affect on measurements recorded. Both Gottschalk [22] and Mayfield [25] have recognized that achieving equal leg length would be the advantageous ideal.

When the X-rays were taken in the two studies is of relative importance. Gottschalk et al.'s study took survey X-rays after limb manufacture, similar to the procedure for the quadrilateral group surveyed by Mayfield. The revised alignment Proto-IC sockets of Mayfield's study were X-rayed during manufacture. Mayfield contributed a chapter titled "Vietnam War Amputees” to the 1994 book Orthopedic Surgery in Vietnam, a text written and revised during the 1970s but published in 1994 [19]. A section in this chapter details the use of the weight-bearing X-ray as standard 
clinical practice at FAMC to ensure proper prosthetic alignment. Mayfield writes,

A radiographic technique was developed at Fitzsimons General Hospital to assess the socket fit and the relative adducted/abducted position of the hip joint on the amputated side. ... It was found that the majority of prostheses were fitted with the amputee's hip in abduction and with poor stump support by the lateral wall of the socket. This alignment placed the hip abductor muscles at a disadvantage and gluteus medius type of gait was present. This X-ray technique was utilized during the prosthetic fitting process to assure proper adduction position of the hip [19].

Figures 9 and $\mathbf{1 0}$ show the initial and final X-rays taken during the prosthesis manufacturing process for two patients at FAMC. The photographs of the initial $\mathrm{X}$-rays were taken during the manufacturing of the prosthesis, at which time any necessary changes required could be attended to. The $\mathrm{X}$-rays show radiographic opaque wire solder positioned along the supe-

(a)

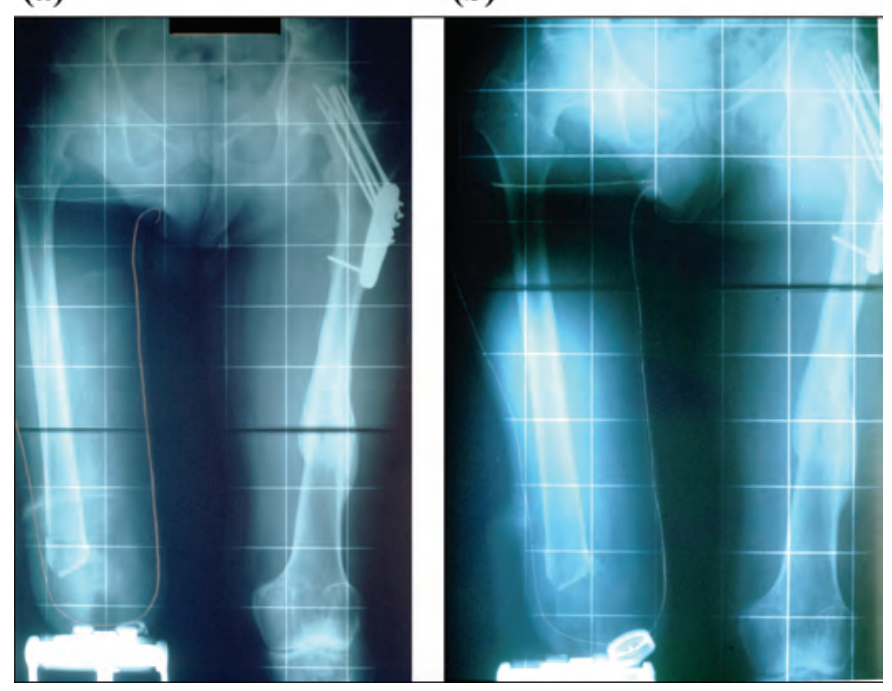

Figure 9.

Fitzsimons Army Medical Center checkout X-rays taken during the prosthesis manufacturing process. (a) Initial X-ray is dated February 19, 1975, and (b) the final X-ray, February 21, 1975. Note increased adduction, improved support for femur, and lateral translation of knee unit in the (b) final X-ray compared with the (a) initial X-ray. Source: http://www.oandplibrary.org/famc/photos/.

rior aspect of the posterior brim and inside the socket bisecting the medial and lateral walls, as per the FAMC X-ray checkout protocol [26]. Photographs showing the placement of the solder wire on the prosthetic socket can be found in Figure 11. In Figure 9, the knee has been translated laterally from its original configuration in the initial X-ray as compared with the final X-ray. A concomitant improvement in adduction of the femur, as well as improved femoral support along the lateral wall of the socket, is also recorded in the final X-ray. In the initial X-ray of Figure 9, a likely pressure point exists between the distal end of the femur and the lateral wall of the socket. This pressure has been remedied by reshaping the lateral wall as seen in the final X-ray. In Figure 10,

(a)

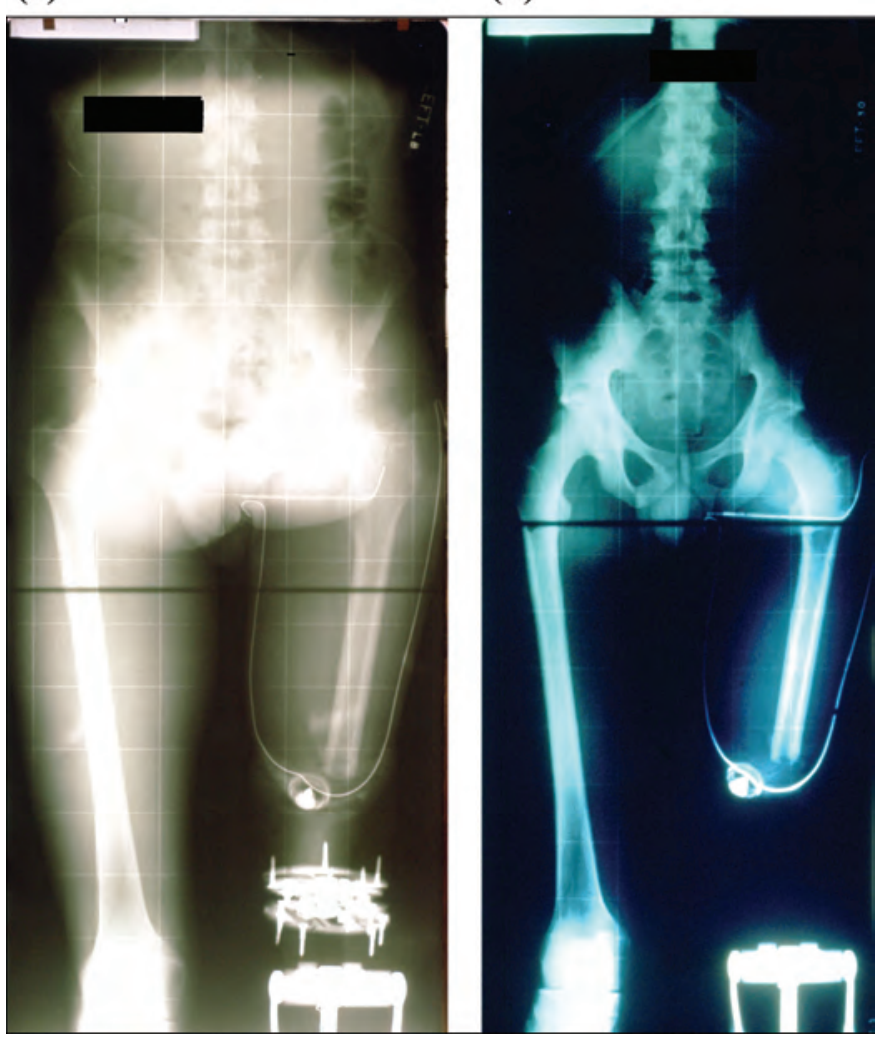

Figure 10.

Fitzsimons Army Medical Center checkout X-rays taken during the prosthesis manufacturing process. (a) Initial X-ray is dated October 16, 1975, and the (b) final X-ray October 29, 1975. Note the temporary Staros-Gardner Adjustable coupling between the knee bolt and socket in the (a) initial X-ray.

Source: http://www.oandplibrary.org/famc/photos/. 

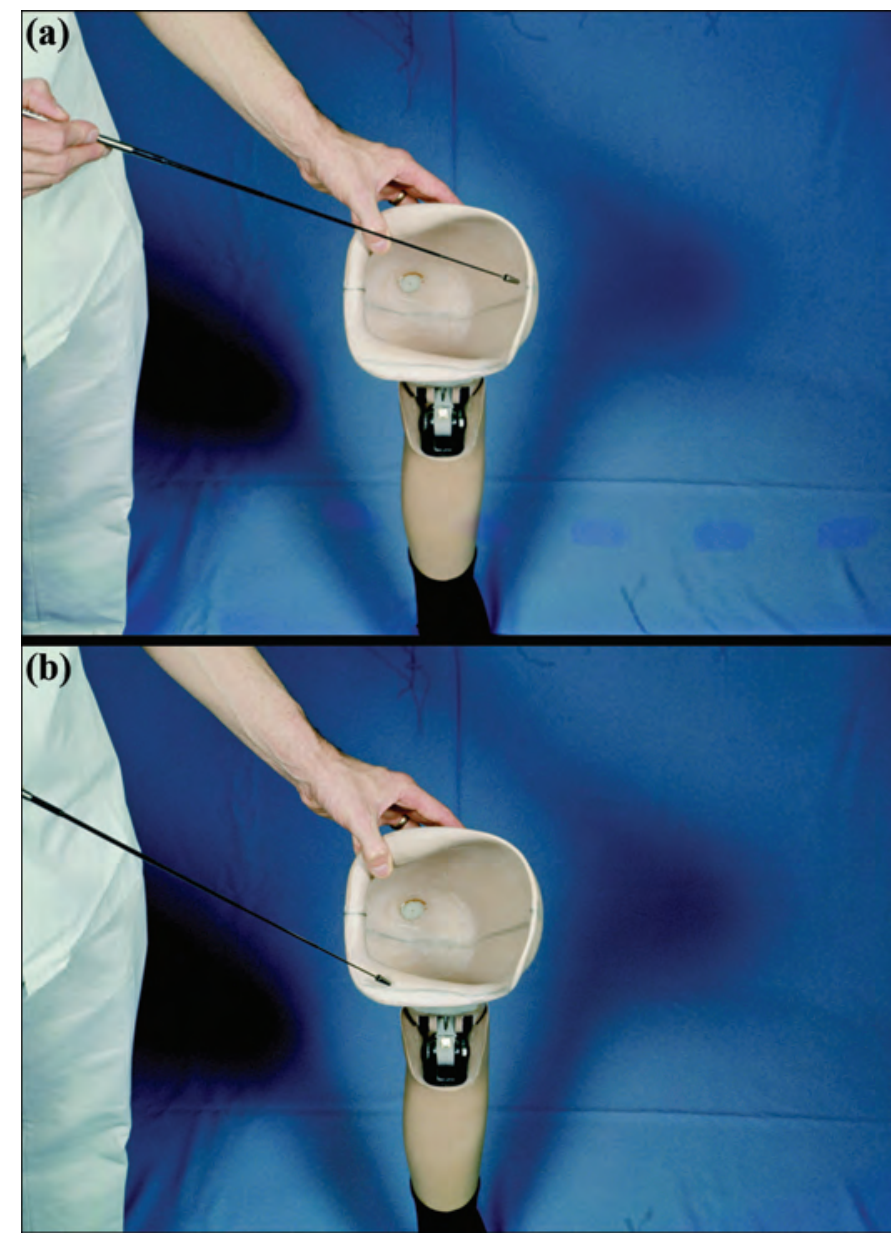

Figure 11.

Photographs showing the placement of radiographic opaque solder wire on a standard quadrilateral transfemoral socket prior to X-ray. (a) Shows the solder wire inside the prosthetic socket bisecting the medial and lateral walls and (b) shows the wire placement on the posterior shelf. Source: http://www.oandplibrary.org/famc/photos/.

the Staros-Gardner Adjustable coupling can be seen in the initial X-ray before transfer of alignment in the final X-ray; slight changes to alignment can be observed.

Beginning in March 1974, the X-ray was an integral part of the prosthetic evaluation process at FAMC [26]. The X-ray checkout consisted of specific points of evaluation. The following six "deviations from traditional alignment criteria" were examined by X-ray as per the recovered FAMC document titled " $\mathrm{X}$-ray evaluation of the above knee socket; A supplement to standard check-out procedures" [26]:

1. Hip Adduction.
2. Lateral Wall Support.

3. Pressure Points.

4. Leg-Length Discrepancy.

5. Ischial Seat.

6. Total Contact.

Between late 1975 and early 1976, FAMC designed a scientific exhibit titled "A look through the above knee prosthesis," which introduced and documented the X-ray checkout of the transfemoral prosthesis [27-28]. The official monochrome Armed Forces Institute of Pathology (AFIP) exhibit photograph is pictured in Figure 12.

The X-ray exhibit was constructed by the AFIP and the narrated slideshow presentation was organized by Scanlon (cited coauthor in Mayfield's research) [27] with staff support of FAMC.* The slides were produced by FAMC's Medical Illustrations Audio Visual Activity, which was located right next to the physical therapy department in the hospital. ${ }^{*}$ The exhibit toured physical therapy meetings [28] and the AOPA Interbor International Congress and Assembly in 1976 [29] and was then returned to the AFIP.

In a letter sent to Mayfield, dated February 13, 1976, Scanlon describes the exhibit:

I prepared a scientific exhibit through AFIP ... The exhibit consisted of a 40 slide presentation with a taped narration explaining how the X-ray is taken and the 7 points we evaluate. I did not go specifically into Ivan's alignment in the exhibit, however many of the slides were on the medio-lateral alignment of the femur within the socket. I also had X-rays on the 2 side panels showing the before and after alignment plus pictures of the old and new prosthesis [28].

The narrated slide presentation was projected on the central display screen of the exhibit. Viewers of the exhibit would pick up one of the earphones and listen while watching the slideshow presentation. The $\mathrm{X}$-ray checkout procedure was introduced and its efficacy supported by the $\mathrm{X}$-rays shown. Seven checkout

* Recorded interview, Jim Scanlon, PT: 08/14/07. 


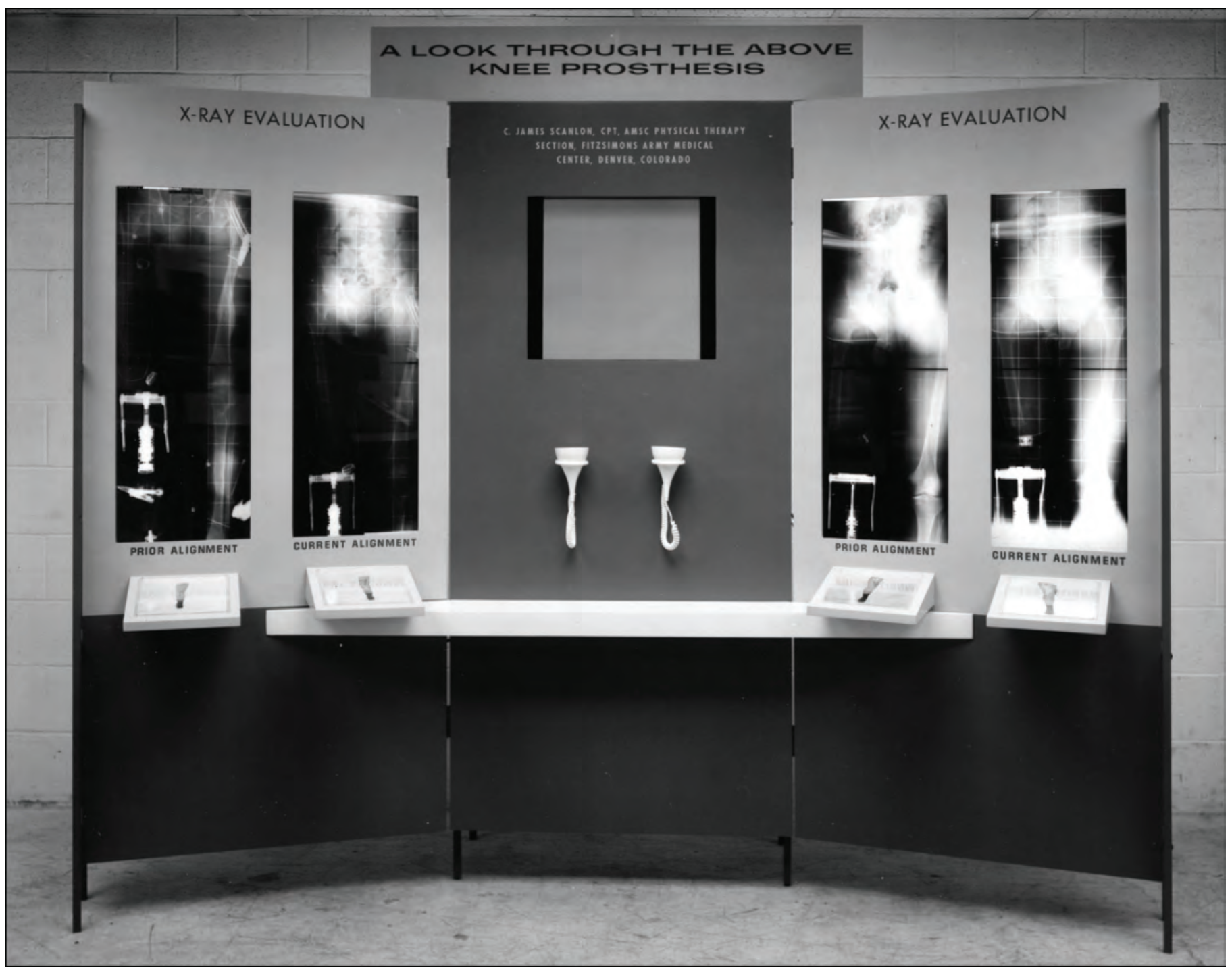

Figure 12.

Armed Forces Institute of Pathology photograph (Negative No. 76-706) depicting Fitzsimons Army Medical Center’s 1976 X-ray exhibit. The exhibit toured several physical therapy and prosthetic meetings during 1976. Source: http://www.oandplibrary.org/famc/?linkto=xrayexhib\#xrayexhib.

points for evaluation were introduced and supported with X-ray examples. The exhibit's slideshow has been carefully reconstructed from surviving $35 \mathrm{~mm}$ slide duplicates. The narration has been rerecorded using the recovered transcript and the slides arranged according to Scanlon's contemporaneous handwritten notes. It can be viewed in an Adobe Flash presentation in the Orthotics and Prosthetics Virtual Library's FAMC Institutional Memory Preservation Project (http://www.oandplibrary.org/famc/). The recovered transcript of this exhibit's slide narration is also available on the Web site [30].
Similar to Mayfield's reference in Orthopedic Surgery in Vietnam, the exhibit documents the use of X-rays during limb manufacture. The exhibit's transcript of slide 36 states, "X-ray pictures are taken before the prosthesis is finished so that deviations from optimal alignment may be recognized and corrected" [30]. The limbs labeled Proto-IC in Mayfield's study were limbs manufactured employing X-ray screening during manufacture. The effectiveness of the $\mathrm{X}$-ray protocol is supported in Mayfield's recovered paper's measurements recording significant adduction for this group. 
Seven points of evaluation, similar to those listed in the recovered FAMC document "X-ray evaluation of the above knee socket; A supplement to standard check-out procedures" [26] are presented as diazo slides in the narrated slideshow. They are framed as questions and listed here:

1. A normal femur is in adduction. Is the amputated femur in adduction?

2. Is the weight properly distributed along the lateral wall?

3. Is the prosthesis the correct length?

4. Is the ischial tuberosity positioned properly over the seat of the prosthesis?

5. Is the ischial seat level?

6. Is the knee bolt level?

\section{Is total contact present?}

The ischial seat deviation from the document " $\mathrm{X}$ ray evaluation of the above knee socket; A supplement to standard check-out procedures" was expanded into two specific examination questions presented in the X-ray exhibit's slideshow. Pressurepoint problems from this document were incorporated into the discussion of lateral wall support in the slideshow. Two discovered outtakes from the exhibit bring the total number of different referenced X-ray points examined at FAMC to nine [31]:

8 . Is the knee bolt $3 / 4$ in. above the medial tibial plateau?

9. Does the distal end of the femur intersect a straight line drawn from the apex of the femoral head through the center of the knee and foot?

The X-ray checkout protocol established by the physical therapy department of FAMC was perhaps the most comprehensive, objective assessment of transfemoral prostheses ever adopted by any institution. The protocol was manifestly a quality assurance program, established for the manufacturing of transfemoral artificial limbs. The X-rays ensured that the design goals of the transfemoral limb were achieved, and design modifications resulted. Had the X-rays in Gottschalk et al.'s study been taken by the staff at FAMC in the mid 1970s, the limbs would have been sent back to the prosthetic shop for revision. Commenting about the reaction to the scientific exhibit during the first meeting at which it was shown, Scanlon wrote in the February 13, 1976, letter to Mayfield that "the response was very good and I was pleased with the comments I received. Many people wanted additional information and asked if this was in the literature so I feel we should continue with our plans to publish an article" [28].

It is Mayfield's recollection, with implications to modern practice, that the X-ray examination, and ultimately the adopted checkout protocol of transfemoral amputees, resulted from a similarity noted in the gait of casualties with united malrotated fractures of the femur [18]. Mayfield relates, "the amount of mal-rotation did correlate with the clinical evidence of gait abnormality . . .” [18]. The prosthetic control of rotation was attempted at FAMC but not successfully achieved. ${ }^{*}$ The significance of this observation is that optimal functional rehabilitation of the transfemoral amputee may be dependent on adduction and limited by rotation as verified by radiographic examination of the hip [32-33].

In the 1950s, when the quadrilateral socket design was coalescing into a standardized design with total contact, suction suspension, and identifying socket shape, early depictions of alignment included the distal femur. Haddan and Thomas depicted a recommended alignment involving the ischial tuberosity, distal femur, knee center, and heel, with lateral wall support of the femur in adduction [34]. In 1952, Canty and Asbelle [35] depicted an alignment that followed the mechanical axis of the lower limb [36]. Canty and Asbelle did not provide specific references to lateral wall support or X-ray verification. The socket style reported by Canty and Asbelle in 1952 had "quadrangular shape" [36], with an open distal end. The design evolved over 7 years as a divergent offshoot into a "closed-end plastic socket” [37], analogous in many ways to the modern transfemoral limb, which employs a prosthetic liner with resulting conical shape. Figure 13 illustrates the

* Recorded interview (Army Prosthetist), Robert Schlesier, CPO: 11/01/07. 
standard alignment of the finalized quadrilateral limb design that first underwent weight-bearing X-ray analysis at FAMC in 1974. The posterior reference line depicted is the accepted quadrilateral alignment line from the ischial tuberosity, resting 1 in. on the posterior shelf of the prosthetic socket to the center of the heel [7]. The ideal adducted position of the femur is illustrated in this diagram, but the distal femur does not intersect the line. Figure 13, which is presented in simplified form, is reproduced from Radcliffe's 1955 article in Artificial Limbs titled "Functional considerations in the fitting of aboveknee prostheses" [4].

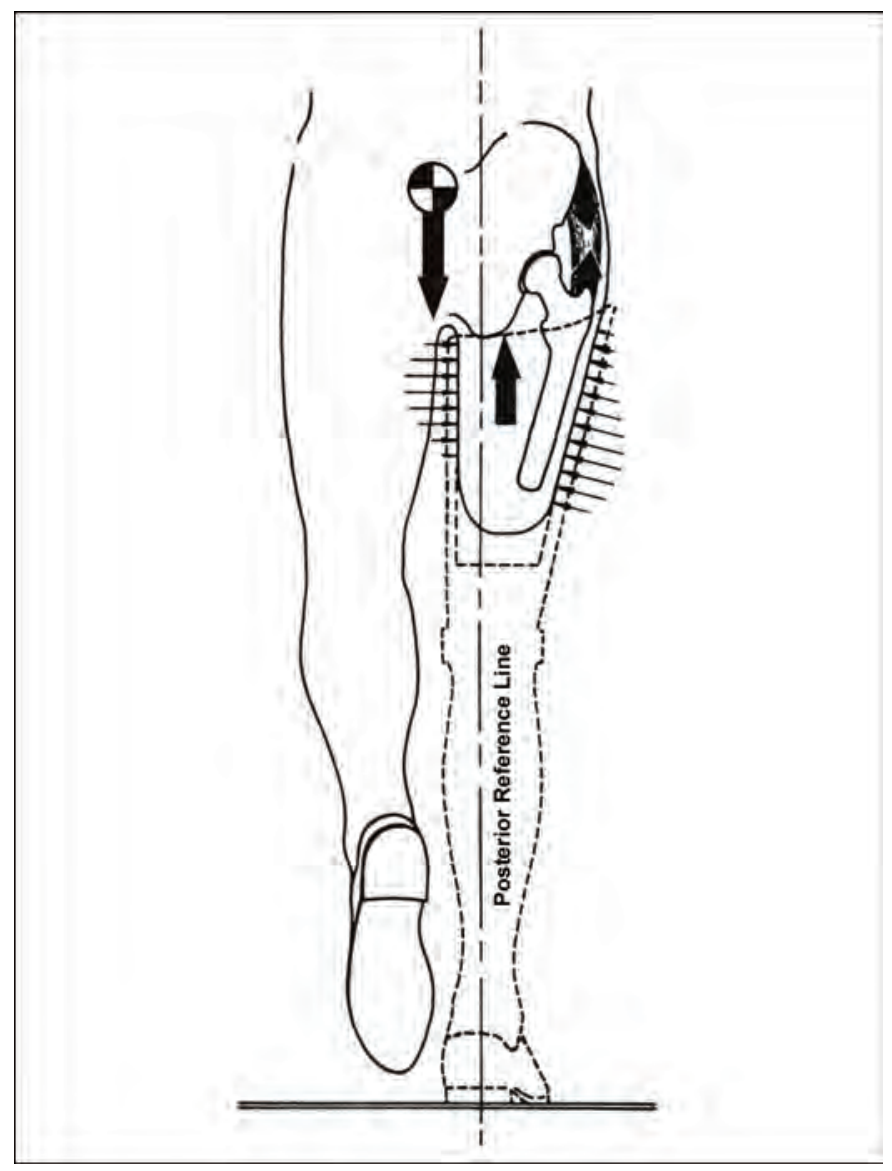

Figure 13.

Standard alignment of the quadrilateral limb design that first underwent weight-bearing X-ray analysis at Fitzsimons Army Medical Center in 1974. Modified from the original source: Radcliffe CW. Functional considerations in the fitting of above-knee prostheses. Artif Limbs. 1955;2(1):35-60. [PMID: 14351063] Available from: http://www.oandplibrary.org/al/1955 01 035.asp.
It was the abduction recorded in a series of weight-bearing X-rays taken on March 13, 1974, of a standard alignment quadrilateral transfemoral limb at FAMC that led to the adoption of the X-ray checkout protocol, ${ }^{*}$ a change in alignment, and eventually socket shape modification of the transfemoral limb. Every modern ischial containment narrow ML socket design can trace its lineage to the two X-rays of a single FAMC patient pictured in Figure 14. X-rays of transfemoral amputees were certainly not unheard of before that time at FAMC ${ }^{\dagger}$ and other military hospitals [28], but these March 13, 1974, X-rays are the ones that inspired Long's alignment protocol [10] and which resulted in the adoption of the X-ray checkout protocol. ${ }^{*}$ The bilateral Xray image in Figure 14(a) first appeared in print as a black-and-white duplication in an article titled, "Normal shape-normal alignment (NSNA) above-knee prosthesis," by Long in 1985 [10]. These Ektachrome color duplications (digitized $35 \mathrm{~mm}$ slides dated December 1975), come from the recovered FAMC X-ray exhibit [38].

Figure 14 shows that the socket has shifted laterally, the ischium rests well above the posterior trimline, and the lateral wall of the socket does not provide the support for the femur. These deviations were not obviously apparent before the X-rays were taken. ${ }^{\ddagger}$ A passage published in 1959 , applicable to these 1974 X-rays, can be found in Clinical Prosthetics for Physicians and Therapists; A Handbook of Clinical Practices Related to Artificial Limbs:

... the amputated femur moves laterally in the mass of tissue in the stump when the gluteus medius contracts, which more than absorbs its excursion and permits the pelvis to tilt excessively, causing pain, and subsequently wide base gait and side-sway ... the thing that must be accomplished is now clear-we must find some way to make the amputated femur stay as nearly as possible in

\footnotetext{
* Recorded interview, Jim Scanlon, PT: 08/14/07.

${ }^{\dagger}$ Personal communication, Jerry Mayfield, MD: 03/05/07.

${ }^{\ddagger}$ Personal communication, Ivan Long, CP: 01/21/07.
} 
the same position it would maintain if it were not amputated. . . . this is accomplished to a large extent by shaping and aligning the socket in such a manner that the stump is held firmly in an adducted position [6, p. 310].

The attempt to support the amputated femur in an adducted position, an established design goal of the quadrilateral socket, resulted in the revised alignment and socket design for the patients at FAMC. It was accomplished almost exactly as described in the quoted passage; the key supplement to the process was the addition of the diagnostic checkout X-ray. Figure $\mathbf{1 4}$ is also an example of Mayfield's observation of the external rotation that occurred in the FAMC amputees. The femoral rem-

(a)

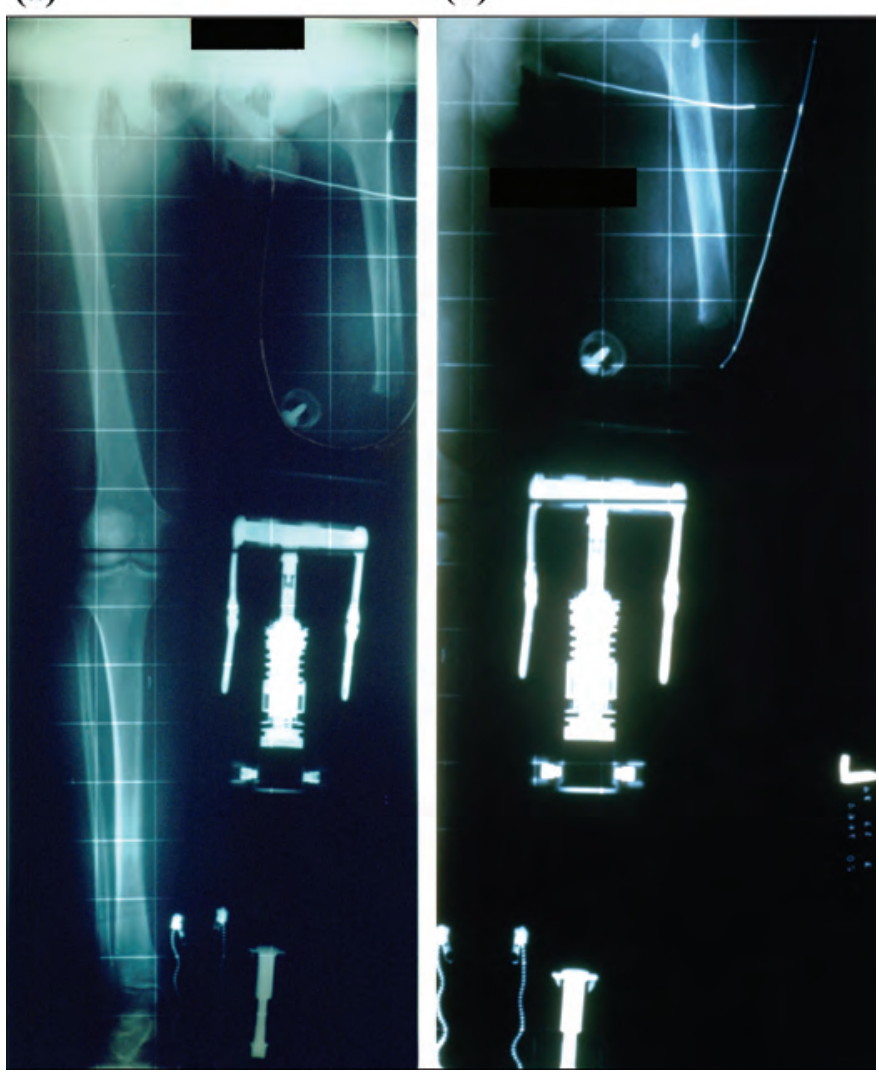

Figure 14.

(a)-(b) X-rays taken on March 13, 1974, of a transfemoral patient at Fitzsimons Army Medical Center showing abduction of the femoral remnant. Long's alignment protocol and FAMC's adoption of X-ray checkout resulted from these X-rays.

Source: http://www.oandplibrary.org/famc/photos/. nant presents a more pronounced lesser trochanter, a less prominent greater trochanter, and a less defined-appearing angle of the femoral neck than the contralateral side [32-33].

In Figure 15, red posterior reference lines of the quadrilateral design have been added to the March 13, 1974, FAMC bilateral X-ray as well as to the patient's replacement limb X-rayed in July 1975. The blue alignment lines are the ninth (outtake) checkout point from the recovered slideshow of the 1976 X-ray exhibit and are examples of Long's Lines from Long's 1975 technical note. Regarding Figure 15, the divergence of the alignment lines is dramatic in a socket whose lateral wall does not provide support of the femur in adduction.

(a)

(b)

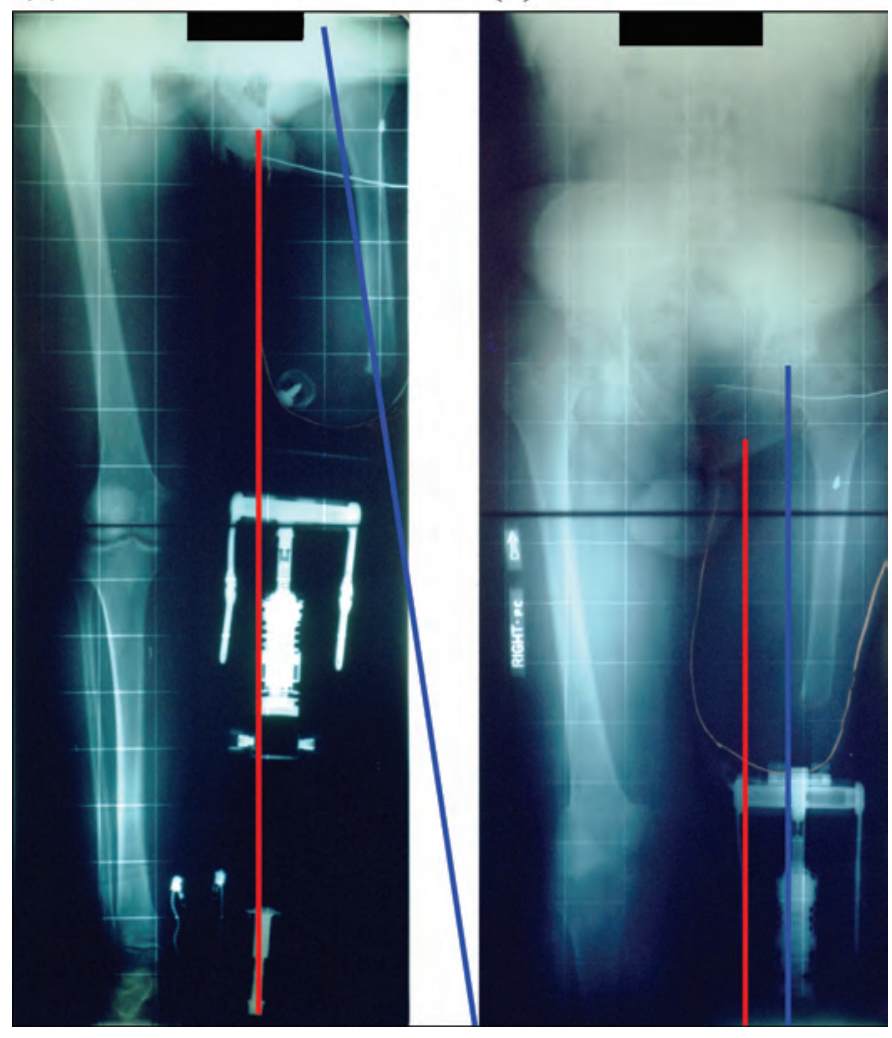

Figure 15.

(a) The quadrilateral posterior reference line (red) and Long's Line (blue) applied to Figure 14(a). (b) These alignment lines are also applied to the patient's replacement limb, X-rayed in July 1975 at Fitzsimons Army Medical Center. Modified from the original source: http://www.oandplibrary.org/famc/photos/. 
Photographs of the prosthetic limbs and their respective X-rays of a FAMC patient have been recovered. This patient was apparently initially fitted with a quadrilateral limb and then provided a replacement revised alignment Proto-IC limb [16]. Figure 16 is a picture of a quadrilateral limb and associated X-ray dated October 1, 1975.

The lateral wall of the pictured limb appears appropriately sloped, but the X-ray reveals abduction of the femur. It is difficult to dismiss the abduction found in the X-ray as being the result of a poorly constructed limb. Figure $\mathbf{1 7}$ is a photograph of a revised alignment Proto-IC limb [21] and associated X-ray. The lateral wall has been reshaped and the socket translated relative to the knee-shin unit. The limbs and X-rays depicted in Figures 16 and $\mathbf{1 7}$ are from the same FAMC patient.

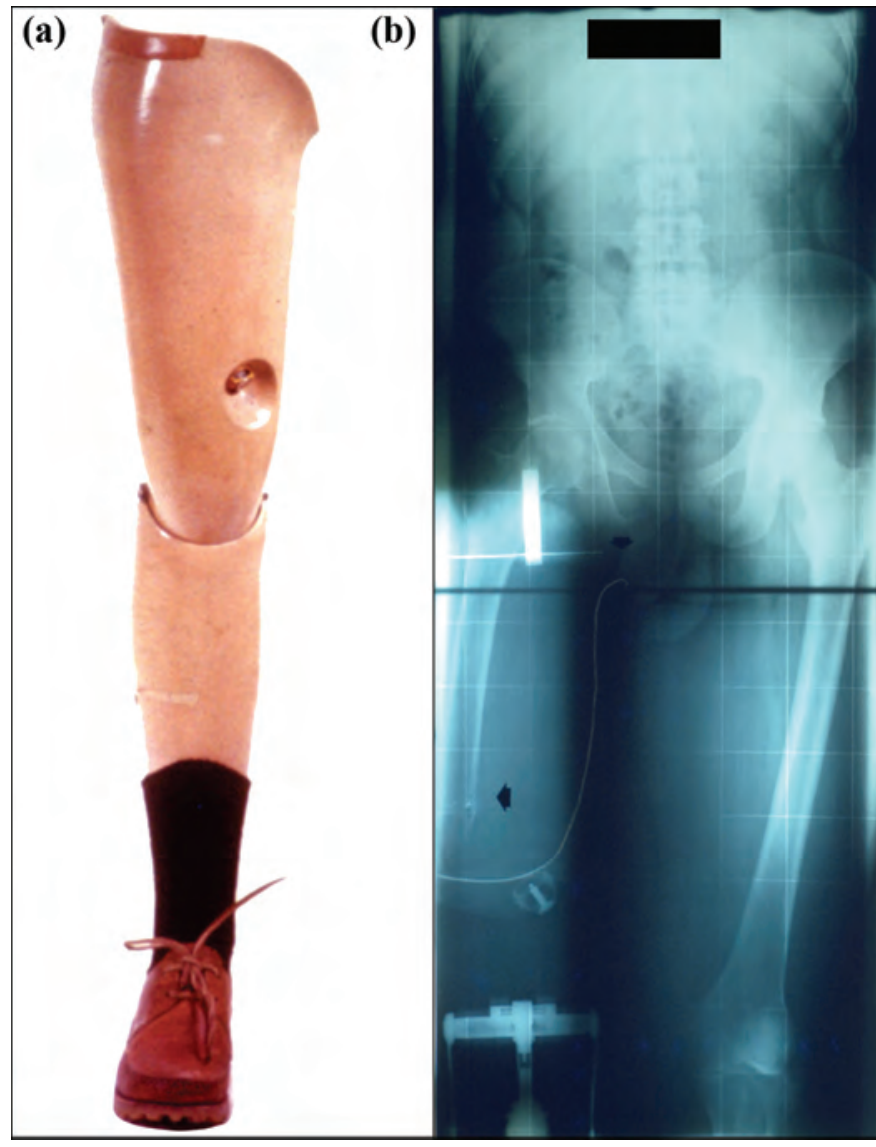

Figure 16.

(a) Quadrilateral limb and (b) associated X-ray dated October 1, 1975. Source: http://www.oandplibrary.org/famc/photos/.
The limb X-rayed in Figure $\mathbf{1 7}$ was made by Long, who in personal communication provided a critique and analysis of his early prosthetic design efforts:

My later sockets show greatly improved support for the femur compared to my first ones. The distal femur must not contact the lateral wall under any circumstance. Plaster must be removed to provide support along the shaft of the femur.

Please keep in mind the limbs made in the seventies were not perfect. Improvements did not come in one giant step. There were no reference books to research. Studying pictures and X-rays was my way of making improvements. The pictures ... . are not to be represented as perfect. I hope they show improvement. $^{\dagger}$

Long was also queried about the unique look of the limb and X-ray pictured in Figure 17. He shared, "What you see is a piece of horsehide covering foam rubber that was added for cosmesis. When I moved the distal socket medially to achieve adduction, there was a concavity in the lateral wall that I built up with foam rubber and covered with horsehide.” checkout weight-bearing X-rays of Vietnam casualties that brought about the changes in the quadrilateral prosthesis design. The modifications undertaken by Long were in accordance with known prosthetic biomechanical design goals but achieved with a unique reduction to practice. The X-ray provided the objective assessment, and a new prosthetic alignment and socket design emerged.

The term "Long's Line" was first coined by FAMC physical therapists [39-40] in describing Long's alignment protocol that used a line that runs directly from the center of the socket, passing through the distal femur to the center of the prosthetic heel [9]. Long adds, "This line is not always plumb but is always straight and should be drawn from the center of the hip joint through the distal femur and down

\footnotetext{
*Personal communication, Ivan Long, CP: 01/13/07.

${ }^{\dagger}$ Personal communication, Ivan Long, CP: 02/03/07.

${ }^{\ddagger}$ Personal communication, Ivan Long, CP: 02/16/07.
} 


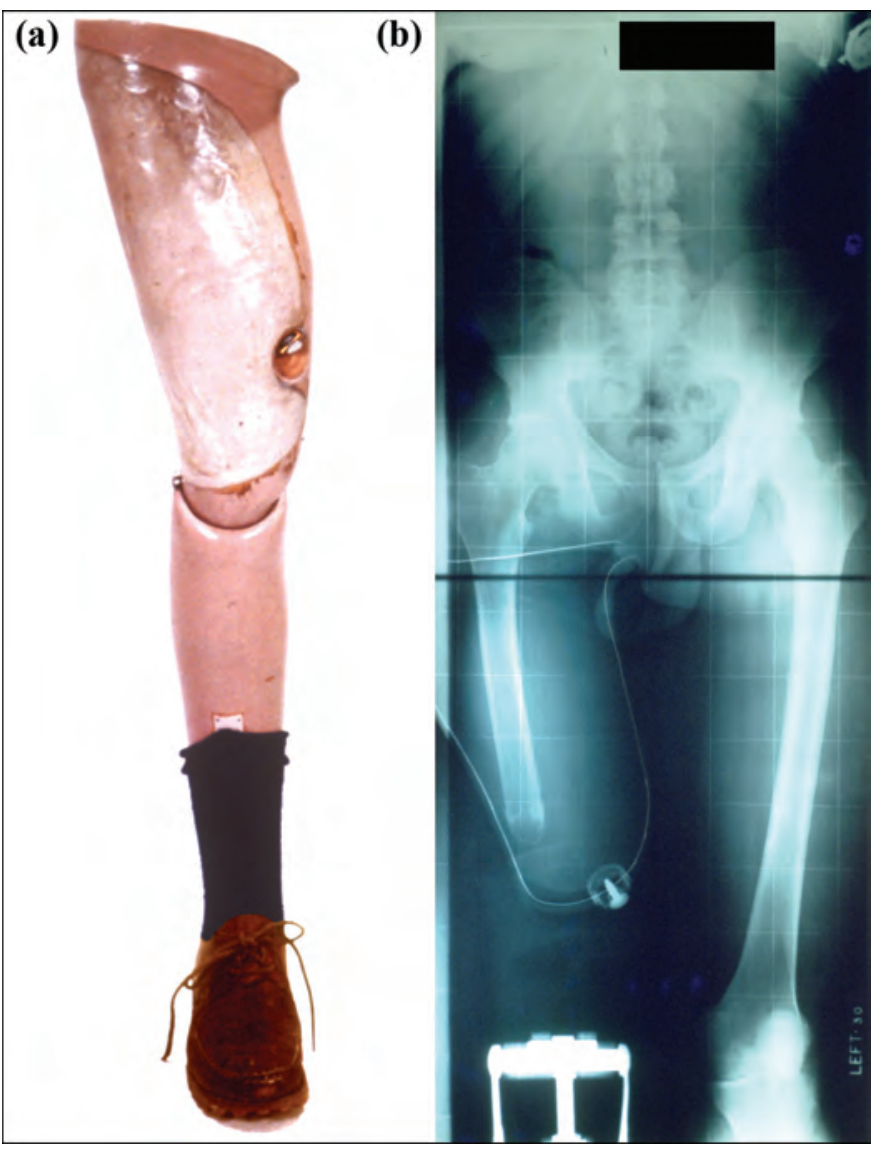

Figure 17.

(a) Protoischial containment limb and (b) associated X-ray dated October 29, 1975. Source: http://www.oandplibrary.org/famc/photos/.

to the center of the foot.”* The revised alignment prostheses referenced in Mayfield's abstract and recovered paper, which have been categorized as Proto-IC in this text, describe transfemoral artificial limbs manufactured using Long's Line. This revised bench alignment also follows the mechanical axis of the leg [36] if the amputated femur was in normal adduction. The objective of using Long's Line is to allow the patient, through prosthetic alignment and socket modification, the ability to achieve anatomical adduction of the femoral remnant [9-10]. It can be understood that objective confirmation of Long's Line is only attained through X-ray. This is reported in the first sentence of Mayfield's abstract: “The degree of hip adduction/abduction of the stump of an

*Personal communication, Ivan Long, CP: 01/21/07.
AK [above-knee] amputee wearing a prosthesis cannot be accurately evaluated by physican [sic] examination" [16]. Figure 18 shows an illustration of Long's Line, which was reproduced from Mayfield's recovered paper [21]. A surviving black and white picture of an "initial" exoskeletal setup, with electrical tape representing Long's Line, has been added to the illustration. The pictured "initial" limb, which has been assembled but not cosmetically finished, would have been X-rayed at this stage of manufacture according to the FAMC protocol. Any changes, if necessary, could have easily been accomplished at this stage of production.

Twenty patients in Mayfield's study were X-rayed in both their standard quadrilateral limb and their replacement Proto-IC limb. These repeated-measure patients are of particular interest and populate a portion of both Groups 1 and 2 in Table 1 . The only change to these 20 patients was a replacement prosthesis employing the revised Long's Line alignment and X-ray screening during manufacture of the artificial transfemoral limb. The descriptive statistics of these 20 patients in Mayfield's study are presented in Table 2 (Microsoft Excel 2002). Figure 19 is a scatter plot of the data from this unique 20-patient subgroup. According to data in Mayfield's recovered research paper, 25 percent of the quadrilateral limbs (5 patients) and 15 percent of the revised alignment Proto-IC legs (3 patients) in this 20patient subgroup are noted as having a leg-length discrepancy [21]. As pointed out in Mayfield's recovered study, prosthesis length discrepancies exaggerate either the abduction or adduction measurements of both limbs depending on whether the prosthesis is too short or too long. According to the sound-side limb measurements found in Mayfield's study, a difference of $0.6^{\circ}$ existed between the sound-side means of the quadrilateral $\left(9.3^{\circ}\right)$ and Proto-IC $\left(8.7^{\circ}\right)$ limbs for these 20 patients who received both styles of limbs [21]. The effect of leg-length differences on adduction in the five quadrilateral and three revised alignment Proto-IC patients is too small to effectively analyze, but given the analyzed sound-side means, this effect appears negligible. The improvement of $8.8^{\circ}$ toward adduction, calculated from the quadrilateral and Proto-IC femoral remnant means displayed in Table 2, achieved in these 20 patients suggests that socket design and alignment do have an influence on the angular position 


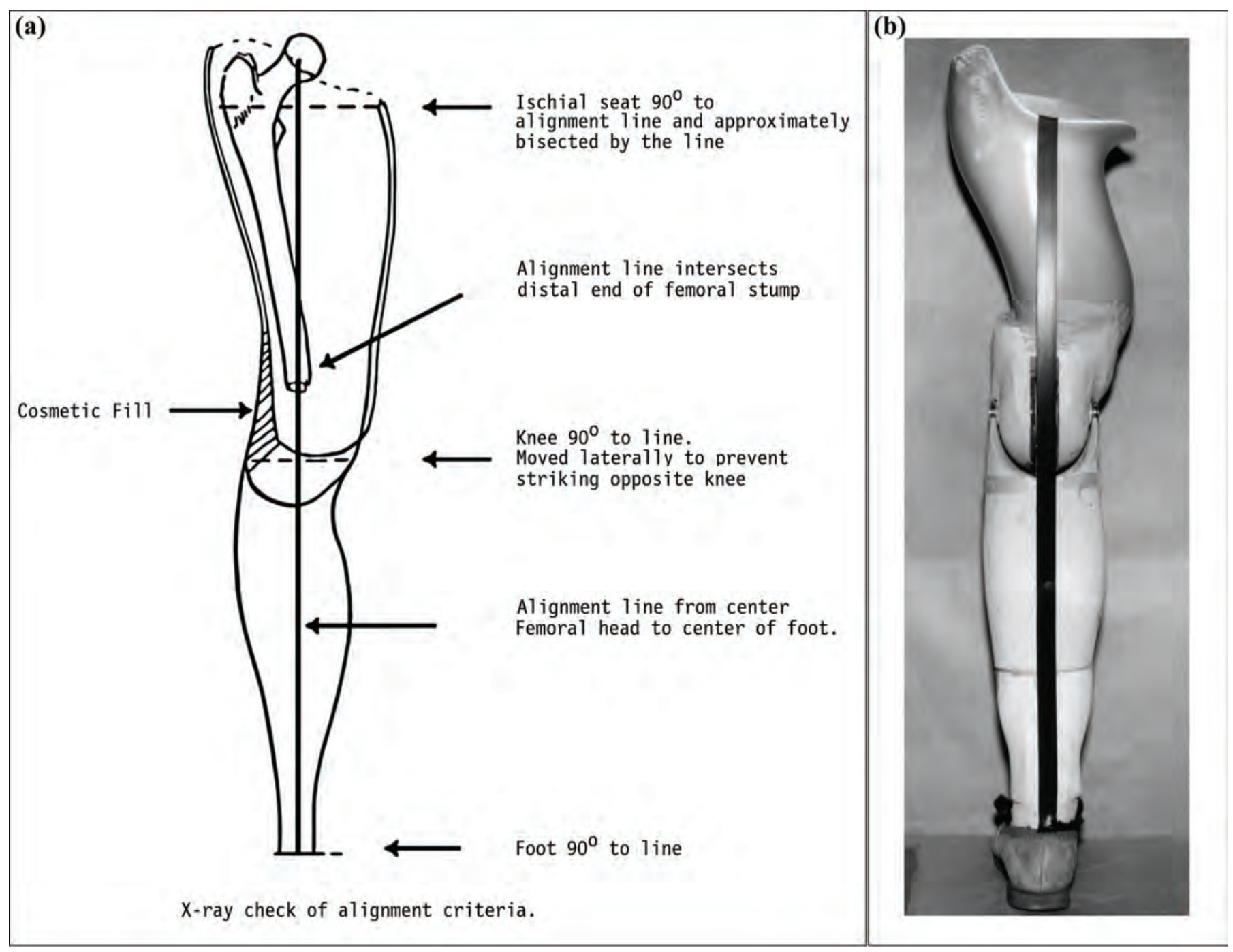

Figure 18.

(a) Long's line is depicted in an illustration from Mayfield's recovered and retypeset research paper, schematically showing how it is used as part of the X-ray checkout. (b) Long's line is applied to a transfemoral prosthesis prior to final finishing. Were an X-ray taken at this stage of manufacture, it would be labeled "initial," and any changes, as determined by X-ray, could be easily accomplished at this stage of production. Modified from the original source: Mayfield GW, Scanlon J, Long I. A new look to and through the above-knee socket (Recovered research paper) [Internet]. Gainesville (FL): Digital Resource Foundation for the Orthotics and Prosthetics Community, The Orthotics and Prosthetics Virtual Library, Fitzsimons Army Medical Center Institutional Memory Preservation Project; 2009 Jun 22 [cited 2009 Jun 30]. Available from: http://www.oandplibrary.org/famc/?linkto=abnewlook\#abnewlook.

of the femoral remnant and further challenges conclusions to the contrary.

At the time of publication of Gottschalk et al.'s 1989 research, the ischial containment socket design had reached its industry peak and competing designs had emerged. A specific Medicare L-code had been established, which encouraged the adoption of this particular style of socket. Although the modern origins of the ischial containment narrow ML socket can be traced to one single patient's X-rays taken at FAMC in 1974, variants of the design as practiced and promoted by different prosthetists had by then emerged and one derived design [11] had become well advertised and would eventually be patented [41]. "Protoischial containment" was a descriptive term chosen to describe prostheses manufactured with X-ray intervention in 
Table 2.

Femoral remnant measurement statistics of Mayfield's repeated measures. Patients receiving replacement revised alignment protoischial containment prosthesis for their original quadrilateral prosthesis $(n=20)$. Positive numbers represent adduction and negative numbers abduction.

\begin{tabular}{lcc}
\hline Variable & $\begin{array}{c}\text { Quadrilateral } \\
\text { (Original Prosthesis) } \\
\text { Femoral Remnant }\end{array}$ & $\begin{array}{c}\text { Protoischial } \\
\text { Containment } \\
\text { (Replacement } \\
\text { Prosthesis) } \\
\text { Femoral Remnant }\end{array}$ \\
\hline Mean & -2.8 & 6.0 \\
Median & -2.0 & 6.0 \\
SD & 4.8 & 3.5 \\
Minimum & -13.0 & -2.0 \\
Maximum & 5.0 & 11.0 \\
\hline SD = standard deviation. & \\
Data source: Mayfield GW, Scanlon J, Long I. A new look to and \\
through the above-knee socket (Recovered research paper) [Internet]. \\
Gainesville (FL): Digital Resource Foundation for the Orthotics and \\
Prosthetics Community, The Orthotics and Prosthetics Virtual Library, \\
Fitzsimons Army Medical Center Institutional Memory Preservation \\
Project; 2009 Jun 22 [cited 2009 Jun 30]. Available from: http:// \\
www.oandplibrary.org/famc/?linkto=newlookre\#newlookre. \\
\hline \hline
\end{tabular}

Mayfield's study. It effectively defines the quadrilateral sockets that had received revised alignment and lateral wall modification and thus resulted in "a new look to" the prosthetic design [21].

In the early FAMC-era documents, prochronism notwithstanding, ischial containment is not mentioned and references are only made to quadrilateral checkout points. In personal communication, Long clarifies, somewhat emphatically, the origin of ischial containment:

This is a true version of how I started using ischial containment in all my sockets. When I first started working with the narrow ML concept, we had a problem with lateral gapping. Ischial containment was the answer to that problem. Ischial containment was never used to adduct the femur. Please keep in mind that the amputee adducts the femur. PERIOD. The lateral wall must stay in close contact to prevent even a small degree of abduction when weight is applied. The slightest lateral movement will result in a shifting of the trunk for balance.*

\footnotetext{
*Personal communication, Ivan Long, CP: 11/31/03.
}

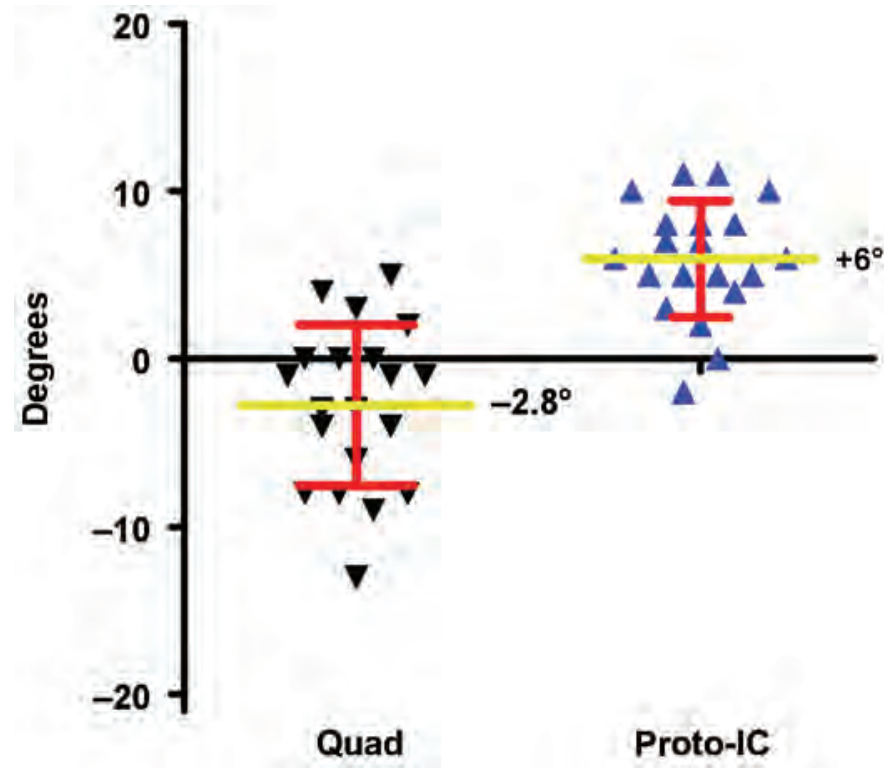

Figure 19.

Scatter plot of transfemoral X-ray femoral remnant adduction/ abduction measurements of the 20 patients in Mayfield's recovered study who received both quadrilateral and replacement protoischial containment (Proto-IC) limbs. Vertical red bands represent standard deviation and bisecting yellow bars indicate the group's mean. X-ray data source: Mayfield GW, Scanlon J, Long I. A new look to and through the above-knee socket (Recovered research paper) [Internet]. Gainesville (FL): Digital Resource Foundation for the Orthotics and Prosthetics Community, The Orthotics and Prosthetics Virtual Library, Fitzsimons Army Medical Center Institutional Memory Preservation Project; 2009 Jun 22 [cited 2009 Jun 30]. Available from: http:// www.oandplibrary.org/famc/?linkto=abnewlook\#abnewlook.

Corroboration of the modern development of ischial containment comes from a recorded interview with John Sabolich, who attributes Long as having brought the concept to his facility in a presentation given in $1981 .^{\dagger}$ Ischial containment is apparent in some of the recovered X-ray images from FAMC. The revised alignment prostheses in Figure 4(b), Figure 10, and Figure 15(b) could be arguably classified as ischial containment sockets. Lead solder was placed along the posterior superior wall of the socket, equally dividing the lateral and medial walls of the socket, as per the FAMC X-ray checkout protocol [26]. It was not placed along the posterior medial corner so the achievement of

\footnotetext{
${ }^{\dagger}$ Recorded interview, John Sabolich, CPO: 10/21/07.
} 
ischial containment is subject to a degree of interpretation. Sabolich was the first to use the term "ischial containment" in print in 1985 [11]. Radcliffe classified the socket design as IRC [23-24].

Between Gottschalk et al.'s and Mayfield's studies, $123 \mathrm{X}$-rays were taken of 101 patients. The total number of patients and cases far eclipses any other similar research study. Although research has been published that compares the quadrilateral and ischial containment sockets [42-43] and studies have imaged the femoral remnant in a prosthetic socket [15,44-45], only two other research articles have been published that have measured the angular orientation of the femoral remnant in comparative socket designs using $\mathrm{X}$-ray measurements with a sample size equal to or greater than five patients. The review of these two studies will be mostly limited to their X-ray analysis of alignment and reports on gait, but it should be noted that both studies exceed the research scope of Mayfield and Gottschalk et al., addressing such issues as metabolic efficiency and patient satisfaction with the respective designs.

In 1989, and just a few months prior to publication of Gottschalk et al.'s article, a research article titled, "The effect of the CAT-CAM above-knee prosthesis on functional rehabilitation," by Flandry et al. was published in the journal Clinical Orthopedics and Related Research [46]. Similar to Mayfield's 20-patient subgroup, who received two different sockets/limbs, Flandry et al.'s research involved five transfemoral amputee patients who wore quadrilateral sockets and received replacement ischial containment sockets with a revised alignment protocol "to determine the effect on ambulatory function” [46]. The X-ray measurement technique was also similar to Mayfield's study as illustrated in Figure 7; the only difference is that the horizontal reference line was placed along the "tips of the ischial tuberosity" [46]. In Flandry et al.'s study, both socket styles were X-rayed and "in three of the five patients, abduction in the quadrilateral socket was converted to adduction" [46]. The measurements reported in Flandry et al.'s study showed an average adduction change of $6.5^{\circ}$ in four of the five patients. Flandry et al. noted that the ischial containment socket for these four patients "performed as theoretical concepts suggested" and that the "roentgenographic data parallel observations previously reported by Mayfield et al.” [46].

Along with four "good fittings," one "poorly aligned" fitting was noted in this study [46]. A single patient, the first case of the series, received an ischial containment socket with $7^{\circ}$ change toward abduction ( $2^{\circ}$ adduction to $5^{\circ}$ abduction). A similar occurrence is recorded in Mayfield's recovered research paper: patient number 43 had $5^{\circ}$ less adduction $\left(13^{\circ}\right.$ adduction to $8^{\circ}$ adduction) in a replacement Proto-IC socket [21]. Remarkably, in Flandry et al.'s study, gait deviations were noted as improved with this "misaligned" ischial containment socket, yet this patient presented "a downgrading of the functional ambulatory level, and subjective rejection" of the new socket replacement [46]. Of the four patients with improved adduction, two increased functional ability and two remained at the same level; regarding the entire group, "most gait deviation[s] initially observed as the subjects walked with their quadrilateral sockets were resolved" by conversion to ischial containment sockets [46]. In Flandry et al.'s study, once X-rays revealed a $7^{\circ}$ change toward abduction in the first patient, greater care was extended in fitting the remaining patients [46]. Although X-rays were not described as being a part of the actual manufacturing process, as was the case in the aggregate results from FAMC $[19,30]$ collected in Mayfield's study [18], the interpretation is that the X-ray analysis in Flandry et al.'s study was a causal factor in the improvement in adduction achieved in the ischial containment sockets. The structure of the research undertaken in Flandry et al.'s study, involving active modification of the alignment and socket design, as well as the improvement in adduction recorded, contrasts with conclusions regarding the lack of socket and alignment influence on the angular position of the femoral remnant.

Modified quadrilateral sockets in Mayfield's study that were X-rayed during manufacture with lateral wall alterations and improved adduction have been classified as Proto-IC in this text. Flandry et al.'s study, with some applied liberty, defined Mayfield's revised alignment sockets as contoured 
adducted trochanteric-controlled alignment method (CAT-CAM) as a means of differentiating the sockets. Extrapolating from Mayfield's and Gottschalk et al.'s research, adduction improvement is not dependent on socket style but on design goals, emphasized and objectively confirmed. Had the quadrilateral sockets been subject to a revision in Flandry et al.'s study, which did not include containment of the ischial tuberosity within the posterior medial border of the prosthetic socket, improved adduction would have still been recorded. The hybrid nature of Flandry et al.'s study, as both an X-ray survey of existing quadrilateral sockets and a study-specific manufacture of CAT-CAM sockets, was an aspect of the study that was called into question in print.

Fourteen months after publication of Flandry et al.'s paper, a letter to the editor was published by the same journal: it was written by Gottschalk, who argued that the research was "flawed in several areas" [22]. Criticism was presented that focused on prosthesis length inequalities, X-ray measurement protocol (both of which would apply to Mayfield's study), lack of statistical analysis, and the age and fit of the analyzed quadrilateral sockets. Although the limb-height influence explanation could be classified as tautological, Flandry and Perry (a coauthor on the original publication) responded and clarified each raised point of contention, noting in particular that the original quadrilateral sockets surveyed were "well fitted" [47]. The two authors dismissively concluded that Gottschalk's arguments were "assumptions and therefore unfounded" [47]. This is about as exciting as it gets in the staid realm of research.

Ten years after publication of both Gottschalk et al.'s and Flandry et al.'s research, a study titled, "Subjective evaluations and objective measurements of the ischial-ramal containment prosthesis” by Hachisuka et al. was published [48]. Six quadrilateral and six IRC sockets were analyzed, and X-rays were used in the study to measure the position of the femoral remnant. Weight-bearing X-rays were remarkably "taken while the subjects were standing on one foot . . . simulating mid-stance of gait” [48]. The X-ray measurement procedure followed protocols similar to Mayfield's study (Figure 7). Abduction was uniquely defined as the involved-side measurement subtracted from the sound side. Eight degrees less abduction was recorded in the ischial containment sockets than the quadrilateral. Hachisuka et al. write, "By X-ray, the stump of the IRC group was maintained significantly more adducted during one foot standing on the prosthesis" [48]. The IRC group involved positive angular measurements that were used to calculate abduction. These measurements would have been reported as adduction in Mayfield's, Flandry et al.'s, and Gottschalk et al.'s studies.

Lateral socket forces were calculated in the study via forceplate measurements and were used to explore the abduction occurrence (as defined) in the IRC sockets X-rayed. Hachisuka et al. write,

... the lateral force toward the amputated side during mid-stance is not significantly less in the IRC group. This means that the IRC socket design is useful for preventing the stump from abduction during the stance phase but is unable to prevent abduction completely. The stump abduction may be affected by an amputee's habitual walking pattern, gluteal medial muscle atrophy, and method of transfemoral amputation [48].

Although the survey of IRC sockets showed abduction as per the study's unique definition, no sockets were modified in the study to explore whether the socket "is unable to prevent abduction completely" [48]. It is not suggested that this conclusion was ever applied to sockets outside the study by the principal investigator, but the limitation is noted. Gottschalk and Still's 1994 biomechanical model [2] of the amputated residual limb is cited in explanation of this abduction occurrence; yet the role of the prosthetic socket, even though the abduction recorded in the IRC limbs is significantly less than the quadrilateral (and would be considered adduction in the other studies), is not explored indepth in the study. Gait was not reported as being improved between the two socket styles [46], so abduction or the severity of abduction would not have been considered to have an effect on improving functional patient outcomes. Nonetheless, the occurrence of a significant difference in the abduction measurements (as defined) does call to question the 
conclusion that the prosthetic socket does not influence the position of the femur.

Figure 20 is a series of X-rays taken of a single patient in three prosthetic limbs over a 14-month period from March 1974 to May 1975. The evolution of the narrow ML socket design is captured in these images. Viewing from left to right, note the improving support for the femur in the latter two sockets X-rayed at FAMC. The last socket on the right (Figure 20(c)) is one of the best recovered $\mathrm{X}$-ray examples of a FAMC patient described in Mayfield's published abstract as being "fitted with . . . revised techniques”: it was made by Long. The socket axis of the limb in Figure 20(c) actually shows less angulation than the socket pictured in Figure 20(b). It also supports the femur fully over the length of the lateral wall. Ischial containment is not revealed in these X-rays; yet the last socket is a Proto-IC socket in that the lateral wall of the socket has been reshaped and presents a revised Long's Line align- ment. These images show a progressive narrowing of the ML aspect of the socket, which was the penultimate evolutionary step to the ischial tuberosity being captured inside the borders of the socket and providing the definition for a new limb/socket category. Recent studies using ultrasound imaging have found abduction occurring during ambulation, increasing to a maximum at midstance [44-45], but it is difficult to conceive of abduction occurring from lateral forces generated during walking in the last recorded evolution of this patient's socket as reduced to practice by Long.

There are 46 limbs in the studies of Mayfield, Flandry et al., and Hachisuka et al. that show significant improvement toward adduction in the prosthetic limb. It is interpreted that socket design and alignment were causal factors in the recorded improvement. These adduction findings, now gathering in magnitude with the recovery of Mayfield's research paper, call to question the conclusion that (a)

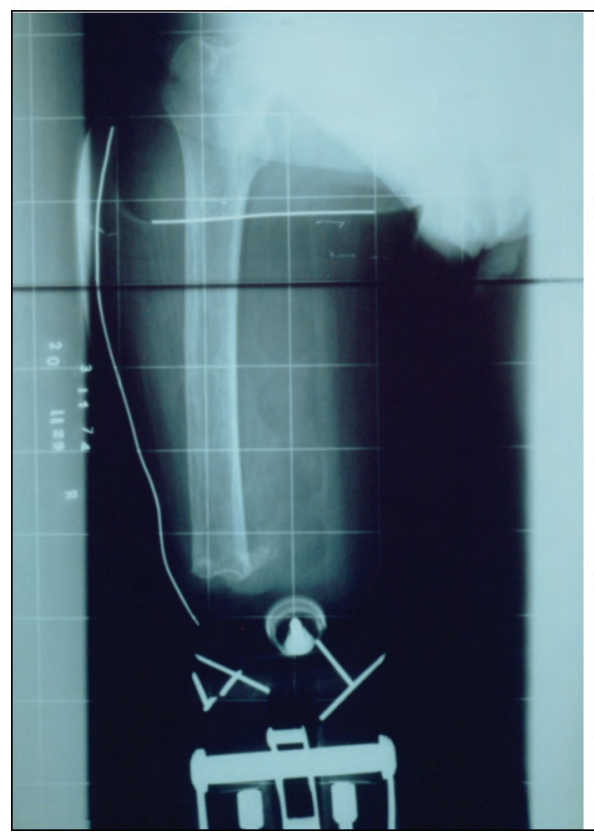

(b)

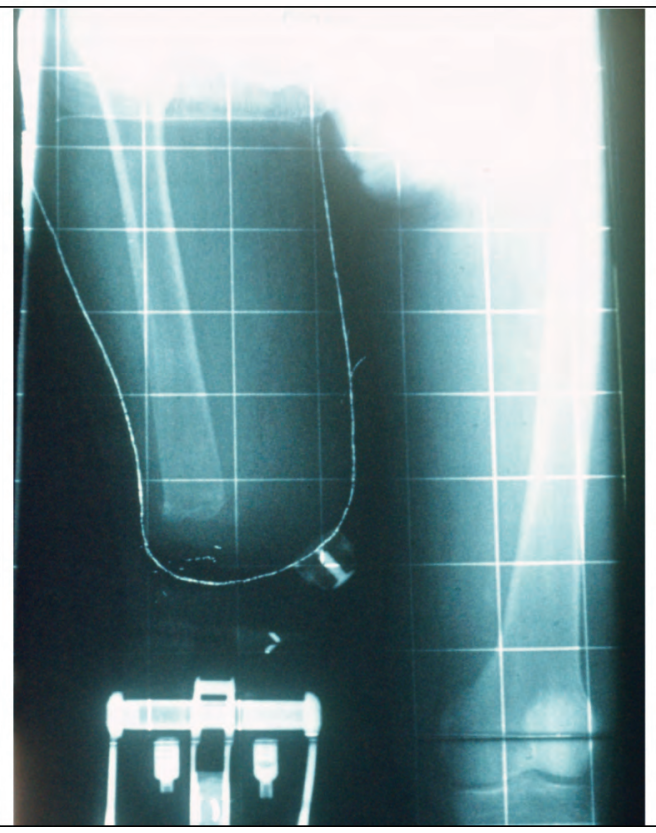

(c)

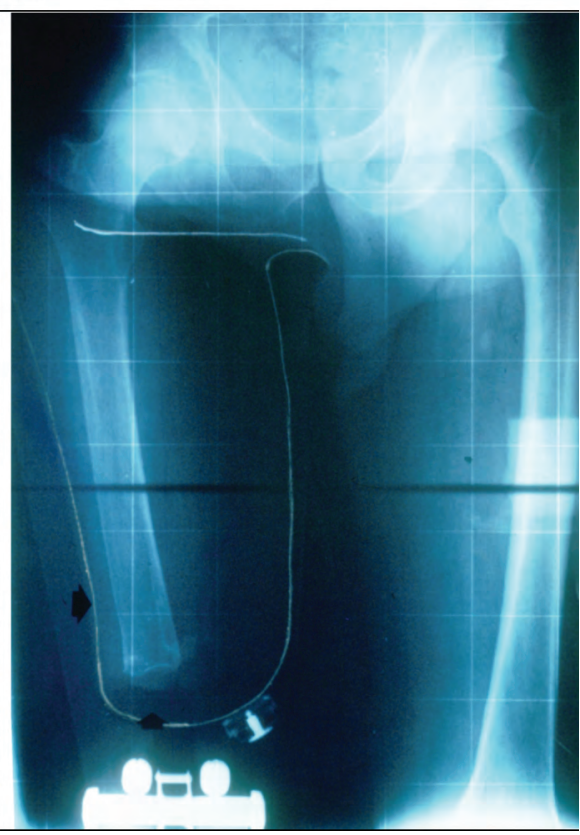

Figure 20.

Evolution of narrow mediolateral (ML) socket as captured on X-ray; same patient depicted in all three X-rays on three different dates over 14 months: (a) March 11, 1974, (b) May 21, 1974, and (c) May 16, 1975. Viewing from left to right, note improving support for femur in latter two sockets X-rayed at Fitzsimons Army Medical Center. These images show progressive narrowing of ML aspect of the socket, which was the second-to-last evolutionary step to the ischial tuberosity being captured inside the borders of the socket and providing the definition for a new limb/socket category. Magnification has been equalized in these X-ray images. Source: http://www.oandplibrary.org/famc/photos/. 
socket design does not influence the position of the femur. The effect on functional outcome for the patient in achieving optimal adduction, as objectively confirmed, remains an area for future investigation because the research findings are contradictory and limited in sample size $[46,48]$. A risk assessment of the ionizing radiation exposure from X-rays will undoubtedly have to be carefully explored and justified in any future X-ray studies. The recovered FAMC documents and reported clinical observations suggest that research exploring the effect of the involved side's hip position-both adduction-abduction and external rotation-on the functional outcome of the transfemoral amputee might well be justified.

Every X-ray survey of transfemoral limbs, with the exception of Hachisuka et al.'s six-patient IRC socket group, which was undertaken without prior $\mathrm{X}$-ray intervention during manufacture or X-ray confirmation immediately after limb manufacture, has revealed abduction of the femoral remnant, with abduction being defined as illustrated in Figure 7. It is unfortunate that Gottschalk et al.'s surgery, with its evident benefits, has been promoted on a conclusion drawn from survey $\mathrm{X}$-rays that revealed unachieved limb-design goals. The conclusion that the femoral remnant is not influenced by socket or alignment conflicts with the research findings of, albeit a limited number, the two modern X-ray studies reviewed. Gottschalk et al.'s 1989 research contains a condemning report related to prosthetic quality as reduced to prosthetic practice. A fundamental contrast point is that when prosthetist Ivan Long was presented with X-ray evidence of femoral abduction, a prosthetic solution was devised. When surgeon Frank Gottschalk discovered femoral abduction, a revised surgical technique was proposed. It would seem that an intelligent synthesis of the two approaches, when possible, would be the beneficial ideal.

In the 20 years since the concept that prosthetic socket and alignment do not influence the position of the femoral remnant was introduced into prosthetic thought, no published critique or consequent study-specific design has appeared in the literature. The unchallenged acceptance of this conclusion has framed the industry's categorization of the ischial containment socket. Because attaining normal adduction in the prosthetic limb is no longer practically regarded as achievable, the ischial containment design has become regarded as an optional socket variant, recommended for patients with a short residual femur length [14] and perhaps employed for issues of comfort [49]. Lost in the acceptance and incorporation of this design has been the objective assessment provided by the diagnostic X-rays that originally brought about the revised alignment and subsequent socket modifications. The adoption of an institutionalized X-ray quality assurance protocol-the specific points analyzed and results obtained over 35 years ago at FAMC - is the forgotten prosthetic legacy of the Vietnam war.

\section{Charles King, CP}

President, Arusha Control, Inc, Cumberland, MD; Partner, O\&P Virtual Library, Digital Resource Foundation for the Orthotics \& Prosthetics Community, Gainesville, FL; Alumnus: Century College Prosthetic Practitioner Program, White Bear Lake, MN

Email: charlesking52@gmail.com

DOI:10.1682/JRRD.2009.07.0092

\section{ACKNOWLEDGMENTS}

Sincere appreciation is extended to the many people who assisted in making this undertaking possible: Jim Scanlon, PT; Ivan Long, CP; Jerry Mayfield, MD; Carole Harper; Jane Shofer; Sam Blate; Peggy Dalton, PhD; J. Berge, MSE; S. Fatone, PhD; G. Klute, PhD; C. Langevin; B. Troxell; T. Kasecamp; J. Shinn; P. Crisman; R. Fisch; P. Prusakowski, CPO; J. M., CPO; F. G., MD; S. C. Yuhasz, PhD; L. Tinker; L. M. Rosenberg; and Echo Pedersen, RN. 


\section{REFERENCES}

1. Gottschalk FA, Kourosh S, Stills M, McClellan B, Roberts J. Does socket configuration influence the position of the femur in above-knee amputation? J Prosthet Orthot. 1989;2(1):94-102. Available from:

http://journals.lww.com/jpojournal/Citation/1989/10000/ Does Socket Configuration Influence the Position.9.a spx.

2. Gottschalk FA, Stills M. The biomechanics of transfemoral amputation. Prosthet Orthot Int. 1994;18(1): 12-17. [PMID: 8084744]

3. Gottschalk FA. Transfemoral amputation. In: Bowker JM, Michael JW, editors. Atlas of limb prosthetics: Surgical, prosthetic, and rehabilitation principles. 2nd ed. St. Louis (MO): Mosby Year Book; 1992. p. 501-7.

4. Radcliffe CW. Functional considerations in the fitting of above-knee prostheses. Artif Limbs. 1955; 2(1):35-60. [PMID: 14351063]

Available from: http://www.oandplibrary.org/al/1955 01035 .asp.

5. Radcliffe CW. Alignment of the above-knee artificial leg. In: Klopsteg PE, Wilson PD, editors. Human limbs and their substitutes: Presenting results of engineering and medical studies of the human extremities and application of the data to the design and fitting of artificial limbs and to the care and training of amputees. New York (NY): McGraw-Hill; 1954. p. 676-92.

6. Anderson MH, Bechotol CO, Sollars RE. Clinical prosthetics for physicians and therapists; A handbook of clinical practices related to artificial limbs. Springfield (IL): Thomas; 1959.

7. Anderson MH, Sollars RE. Prosthetic principlesAbove-knee amputations. Springfield (IL): Thomas; 1960.

8. American Academy of Orthopaedic Surgeons. Orthopaedic appliances atlas. Vol. 2, Artificial limbs. Ann Arbor (MI): Edwards; 1960.

9. Long I. Allowing normal adduction of femur in aboveknee amputations. Orthot Prosthet. 1975; 29(4):53-54. Available from:

http://www.oandplibrary.org/assets/pdf/

Technical Note, Ivan Long Orthotics and Prosthetic s_December_1975_ocred.pdf.

10. Long IA. Normal shape-normal alignment (NSNA) above-knee prosthesis. Clin Prosthet Orthot. 1985; 9(4):9-14. Available from:

http://www.oandplibrary.org/cpo/1985 04 009.asp.

11. Sabolich J. Contoured adduction trochantericcontrolled alignment method (CAT-CAM): Introduction and basic principles. Clin Prosthet Orthot. 1985;
9(4):15-26. Available from:

http://www.oandplibrary.org/cpo/1985 04 015.asp.

12. King C, McClellan B. Interview: Bruce "Mac" McClellan, CPO [Internet]. Gainesville (FL): Digital Resource Foundation for the Orthotics and Prosthetics Community, The Orthotics and Prosthetics Virtual Library, Reference Section; 2007 Oct 17 [cited 2009 Jun 22]. Available from: http://oandplibrary.org/ reference/interview-mcclellan.asp.

13. Journal of Prosthetics and Orthotics. Front matter: Table of contents. J Prosthet Orthot. 1989;2(1):2.

14. Huston C, Dillingham TR, Esquenazi A. Rehabilitation of the lower limb amputee. In: Dillingham TR, Belandres PV, editors. Vol. 1, Rehabilitation of the injured combatant. Washington (DC): U.S. Army, Borden Institute, Walter Reed Army Medical Center; 1998. p. 79-159.

15. Kahle JT. A case study using fluoroscope to determine the vital elements of transfemoral interface design. J Prosthet Orthot. 2002;14(3):121-26.

DOI:10.1097/00008526-200209000-00007

Available from:

http://www.oandp.org/jpo/library/2002 03 121.asp.

16. Mayfield GW, Scanlon J, Long I. A new look to and through the above knee socket. Orthop Trans. 1977;1(1):95. Available from:

http://www.oandplibrary.org/assets/pdf/

Abstract,_Dr. Mayfield Orthopaedic Transactions, May 1977 ocred.pdf.

17. American Academy of Orthopaedic Surgeons. Final Program [Internet]. Chicago (IL): American Academy of Orthopaedic Surgeons; 1977. [cited 2009 Jun 22]. Available from: http://www.oandplibrary.org/ assets/pdf/Mayfield aaos meeting.pdf.

18. Mayfield G. Recollections of Fitzsimons Army Hospital and the origin of the X-ray checkout of the above knee limb [Internet]. Gainesville (FL): Digital Resource Foundation for the Orthotics and Prosthetics Community, The Orthotics and Prosthetics Virtual Library, Fitzsimons Army Medical Center Institutional Memory Preservation Project: Introductory Letters of Clinical Practice, 2009 Dec 31 [cited 2010 Jan 7]. Available from: http://www.oandplibrary.org/ assets/pdf/letter_from Jerry_W_Mayfield-reset.pdf.

19. Mayfield GW. Vietnam war amputees. In: Burkhalter WE, Ballard A, editors. Orthopedic surgery in Vietnam. Washington (DC): Office of the Surgeon General and Center of Military History, U.S. Army; 1994. p. 130-53.

20. Mayfield, Gerald W. (Acting Chief, Orthopedic Service, Tripler Army Medical Center, Honolulu, HI). 
Letter to: James Scanlon (Physical Therapy Section, Fitzsimons Army Medical Center, Denver, CO). 1976 Nov 4. 2 leaves. Available from:

http://www.oandplibrary.org/assets/pdf/

Mayfield to Scanlon 1976 Nov 4.pdf.

21. Mayfield GW, Scanlon J, Long I. A new look to and through the above-knee socket (Recovered research paper) [Internet]. Gainesville (FL): Digital Resource Foundation for the Orthotics and Prosthetics Community, The Orthotics and Prosthetics Virtual Library, Fitzsimons Army Medical Center Institutional Memory Preservation Project; 2009 Jun 22 [cited 2009 Jun 30]. Available from:

http://www.oandplibrary.org/assets/pdf/

A NEW LOOK TO AND THROUGH THE ABO VE KNEE SOCKET033 ocred-retypeset.pdf.

22. Gottschalk FA. Letter to the editor. Clin Orthop Relat Res. 1990;255:310-11.

23. Schuch CM. Report from: International workshop on above-knee fitting and alignment techniques. Clin Prosthet Orthot. 1988;12(2):81-98. Available from: http://www.oandplibrary.org/cpo/1988 02 081.asp.

24. Pritham CH. Workshop on teaching materials for above-knee socket variants. J Prosthet Orthot. 1988; 1(1):50-67. Available from: http://journals.lww.com/ jpojournal/Citation/1988/10000/

Workshop On Teaching Materials for Above Kn ee.11.aspx.

25. Mayfield, Gerald W. (Acting Chief, Orthopedic Service, Tripler Army Medical Center, Honolulu, HI). Letter to: James Scanlon (Physical Therapy Section, Fitzsimons Army Medical Center, Denver, CO). 1975 Sep 15. 2 leaves. Available from:

http://www.oandplibrary.org/assets/pdf/

Mayfield to Scanlon 1975 Sep 15.pdf.

26. Scanlon J, Doctor B. X-ray evaluation of the above knee socket; A supplement to standard check-out procedures [Internet]. Gainesville (FL): Digital Resource Foundation for the Orthotics and Prosthetics Community, The Orthotics and Prosthetics Virtual Library, Fitzsimons Army Medical Center Institutional Memory Preservation Project; 2009 Jun 22 [cited 2009 Jul 1]. Available from:

http://www.oandplibrary.org/assets/pdf/

Supplement to Standard Check-out Procedureslow resolution.pdf.

27. Pfeiffer, Violet R. (Chief, Physical Therapy Section, Fitzsimons Army Medical Center). Memo to: Chief, Professional Services. 1975 Oct 9. 2 leaves. Available from: http://www.oandplibrary.org/assets/pdf/ Pfeiffer to Professional Services 10-09-1975.pdf.
28. Scanlon, James. (AMSC, Fitzsimons Army Medical Center, Denver, CO). Letter to: Gerald Mayfield (Orthopedic Service, Tripler Army Medical Center, Honolulu, HI). 1976 Feb 13. 1 leaf. Available from: http://www.oandplibrary.org/assets/pdf/ Scanlon to Mayfield, 1976 Feb 13.pdf.

29. American Orthotic and Prosthetic Association. Almanac: Seventh International Congress in Orthotics and Prosthetics: Interbor International Congress and Assembly [Internet]. Washington (DC): American Orthotic and Prosthetic Association; 1976 Oct 19-23 [Cited 2009 Jul 3]. Available from: http://www.oandplibrary.org/assets/pdf/ AOPA Almanac 1976.pdf.

30. Fitzsimons Army Medical Center. Transcript: A look through the above knee prosthesis [Internet]. Gainesville (FL): Digital Resource Foundation for the Orthotics and Prosthetics Community, The Orthotics and Prosthetics Virtual Library, Fitzsimons Army Medical Center Institutional Memory Preservation Project; 2009 Jul 3 [cited 2009 Jul 3]. Available from: http://www.oandplibrary.org/assets/pdf/ AFIP slideshow transcript PDF.pdf.

31. Fitzsimons Army Medical Center. Outtakes: A look through the above knee prosthesis [Internet]. Gainesville (FL): Digital Resource Foundation for the Orthotics and Prosthetics Community, The Orthotics and Prosthetics Virtual Library, Fitzsimons Army Medical Center Institutional Memory Preservation Project: Support Documents; 2009 Jul 3 [cited 2009 Jul 3]. Available from:

http://www.oandplibrary.org/famc/supportdocuments/ alook-outtakes/.

32. Mayfield G. Illustrations provided by Dr. Mayfield, 02/14/2007 [Internet]. Gainesville (FL): Digital Resource Foundation for the Orthotics and Prosthetics Community, The Orthotics and Prosthetics Virtual Library, Fitzsimons Army Medical Center Institutional Memory Preservation Project: Modern Correspondence; 2007 Feb 14 [cited 2009 Jul 3]. Available from: http://www.oandplibrary.org/assets/ pdf/Mayfields 2-14-07 drawings.pdf.

33. Mayfield G. Mal-rotation Union (01-23-07) \& Hip Rotation Diagram (09-30-07) [Internet]. Gainesville (FL): Digital Resource Foundation for the Orthotics and Prosthetics Community, The Orthotics and Prosthetics Virtual Library, Fitzsimons Army Medical Center Institutional Memory Preservation Project: Introductory Letters of Clinical Practice, 2010 Jan 8 [cited 2010 Jan 8]. Available from: 
http://devel.oandpdigital.com/op/oandplibrary-org/ assets/pdf/Mayfield-Malrotation Union.pdf.

34. Haddan CC, Thomas A. Status of the above-knee suction socket in the United States. Artif Limbs. 1954; 12:29-39. [PMID: 13181751]

35. Canty TJ, Asbelle CC. Suction socket prostheses for above knee amputations: Final technical report. Oakland (CA): Artificial Limb Department, Naval Prosthetic Research Laboratory; 1952 Oct 10.

36. Thomas A, Haddan CC. Amputation prosthesis: Anatomic and physiologic considerations, with principles of alignment and fitting designed for the surgeon and limb manufacturer. Philadelphia (PA): J. B. Lippincott Co; 1945.

37. Canty TJ. Construction, fitting and alignment manual for the U.S. Navy soft closed-end plastic above-knee socket. Oakland (CA): Navy Prosthetic Research Laboratory; 1959.

38. Fitzsimons Army Medical Center. Slideshow: A look through the above knee prosthesis [Internet]. Gainesville (FL): Digital Resource Foundation for the Orthotics and Prosthetics Community, The Orthotics and Prosthetics Virtual Library, Fitzsimons Army Medical Center Institutional Memory Preservation Project; 2009 July 3 [cited 2009 Jul 4]. Available from: http://www.oandplibrary.org/famc/alook/.

39. Long I. Normal shape normal alignment (NSNA) [Fitzsimons Edit] [Internet]. Gainesville (FL): Digital Resource Foundation for the Orthotics and Prosthetics Community, The Orthotics and Prosthetics Virtual Library, Fitzsimons Army Medical Center Institutional Memory Preservation Project: Support Documents; 1986 (slides), 2008 (audio) [cited 2009 Jul 5]. Available from: http://www.oandplibrary.org/ famc/supportdocuments/nsna/.

40. Scanlon, Cornelius J. (AMSC, Physical Therapy Section, Fitzsimons Army Medical Center, Denver, CO). Letter to: Gerald Mayfield (Orthopedic Service, Tripler Army Medical Center, Honolulu, HI). 1975 Jul 3. 2 leaves. Available from: http://www.oandplibrary.org/assets/pdf/ Scanlon_to_Mayfield_1975_Jul_3.pdf.
41. Sabolich JA, inventor; Sabolich, Inc, assignee. Artificial limb with anatomically-configured socket. United States patent US 5,246,464. 1993 Sep 21.

42. Gailey RS, Lawrence D, Burditt C, Spyropoulos P, Newell C, Nash MS. The CAT-CAM socket and quadrilateral socket: A comparison of energy cost during ambulation. Prosthet Orthot Int. 1993;17(2): 95-100. [PMID: 8233775$]$

Available from:

http://www.oandplibrary.org/poi/1993 02 095.asp.

43. Hall MG, Fleming HE, Spence WD, Solomonidis SE, Paul JP. Comparative evaluation of the ischial containment and quadrilateral sockets [abstract]. Gait Posture. 1995;3(3):176.

DOI:10.1016/0966-6362(95)99090-8

44. Convery P, Murray KD. Ultrasound study of the motion of the residual femur within a trans-femoral socket during gait. Prosthet Orthot Int. 2000;24(3): 226-32. [PMID: 11195358] DOI:10.1080/03093640008726552

45. Convery P, Murray KD. Ultrasound study of the motion of the residual femur within a trans-femoral socket during daily living activities other than gait. Prosthet Orthot Int. 2001;25(3):220-27.

[PMID: 11860096] DOI:10.1080/03093640108726605

46. Flandry F, Beskin J, Chambers RB, Perry J, Waters RL, Chavez R. The effect of the CAT-CAM aboveknee prosthesis on functional rehabilitation. Clin Orthop Relat Res. 1989;239:249-62.

[PMID: 2912627]

47. Flandry F, Perry J. Reply to Gottschalk. Clin Orthop Relat Res. 1990;255:311-12.

48. Hachisuka K, Umezu Y, Ogata H, Ohmine S, Shinkoda K, Arizono H. Subjective evaluations and objective measurements of the ischial-ramal containment prosthesis. J UOEH. 1999;21(2):107-18. [PMID: 10434359]

49. Visser-Meily JMA, Cluitrans JJM, Deckers JHM, Ring S. Experiences of above-knee amputees with an NML-Socket in comparison with a quadrilateral socket. J Rehabil Sci. 1992;5(1):22-25. 